\title{
Potential Spaces on Lie Groups
}

\author{
Tommaso Bruno, Marco M. Peloso, and Maria Vallarino
}

Dedicated to Fulvio Ricci on the occasion of his 70th birthday.

\begin{abstract}
In this paper we discuss function spaces on a general noncompact Lie group, namely the scales of Triebel-Lizorkin and Besov spaces, defined in terms of a sub-Laplacian with drift. The sub-Laplacian is written as the (negative) sum of squares of a collection of left-invariant vector fields satisfying Hörmander's condition. These spaces were recently introduced by the authors. In this paper we prove a norm characterization in terms of finite differences, the density of test functions, and related isomorphism properties.
\end{abstract}

Keywords Lie groups · Besov spaces · Triebel-Lizorkin spaces

All authors were partially supported by the grant PRIN 2015 Real and Complex Manifolds: Geometry, Topology and Harmonic Analysis, and are members of the Gruppo Nazionale per l'Analisi Matematica, la Probabilità e le loro Applicazioni (GNAMPA) of the Istituto Nazionale di Alta Matematica (INdAM). T. Bruno was also supported by the Research Foundation - Flanders (FWO) through the postdoctoral grant 12ZW120N.

\footnotetext{
T. Bruno

Dipartimento di Scienze Matematiche "Giuseppe Luigi Lagrange”, Dipartimento di Eccellenza 2018-2022, Politecnico di Torino, Torino, Italy

Present Address: Department of Mathematics: Analysis, Logic and Discrete Mathematics, Ghent University, Ghent, Belgium

e-mail: tommaso.bruno@ugent.be

M. M. Peloso (四)

Dipartimento di Matematica, Università degli Studi di Milano, Milano, Italy

e-mail: marco.peloso@unimi.it

M. Vallarino

Dipartimento di Scienze Matematiche "Giuseppe Luigi Lagrange", Dipartimento di Eccellenza 2018-2022, Politecnico di Torino, Torino, Italy

e-mail: maria.vallarino@polito.it
} 


\section{Introduction}

The theory of function spaces, regularity of integral operators, and of solutions of differential equations, began in the setting of Euclidean spaces, with smoothness measured in terms of Sobolev and Lipschitz norms, see e.g. [46]. A. Calderón and A. Zygmund developed the theory of singular integrals, proving their boundedness in the Lebesgue spaces, as well as regularity of solutions of classical differential equations, such as the Dirichlet and Neumann problems, in the case of a half-space and of smooth domains. Among the operators studied were the singular integrals, hence in particular the Hilbert and Riesz transforms, the Poisson integral, and the heat propagator. It is worth noticing that the singularities of the integral kernels of such operators, or better, of the level sets of their moduli, were naturally described in terms of the underlying Euclidean geometry. Such theory then included embedding and interpolation results for Lebesgue, Sobolev and Lipschitz spaces, see e.g. [3]. In this analysis, the Fourier series and transform played a crucial role, and a noticeable application of such techniques was the decomposition initially introduced by Littlewood and Paley, and later developed in depth by E. M. Stein [47]. The Littlewood-Paley decomposition was initially intended to provide a substitute for the Plancherel formula to the $L^{p}$-norms, with $p \neq 2$, but proved to be an invaluable tool in many other situations. The function spaces that naturally arose in studying the regularity properties of aforementioned operators were indeed, besides the Lebesgue spaces, the Sobolev and Lipschitz spaces, and also the Besov spaces. It became then natural to obtain other characterizations for such norms, and in this setting the Littlewood-Paley decomposition proved to be very useful, and was also used to define another, related, scale of spaces, the so-called Triebel-Lizorkin spaces, see e.g. [53], which include the Sobolev spaces as a special case.

While such theory was in its full development, L. Hörmander produced two breakthrough results, [25] and [26]. In [25] Hörmander extended a previous result by Mihlin, developing the theory of $L^{p}$-multipliers of the Laplacian. This approach also stimulated the study of a class of operators that naturally appear while solving partial differential equations involving the Laplacian - for instance the wave equation in the Euclidean space $\mathbb{R}^{d}$.

In [26] Hörmander showed that operators that are sum of squares of vector fields whose commutators up to a finite order span all directions of $\mathbb{R}^{d}$, although non-elliptic, enjoy many interesting and strong properties of elliptic operators, in particular hypoelliticity. Such phenomenon appeared for instance in the case of the Kohn-Laplacian on the boundary of the Siegel upper half-space in $\mathbb{C}^{d+1}$, in the works of A. Korányi and S. Vági [30], J. J. Kohn [28] and, with most relevance to this discussion and the present work, of G. B. Folland and Stein [13]. The operators that were considered in [13], that is the Kohn-Laplacian, the sub-Laplacian, the so-called Folland-Stein operators, their fundamental solutions, or the relative fundamental solutions in some cases, had the singularity that could be described in terms of a different underlying geometry. The boundary of the Siegel upper half-space can be identified with the Heisenberg group, and such geometry was more efficiently 
described using the nilpotent Lie group structure of the Heisenberg group. As a metric space, the Heisenberg group $\mathbb{H}_{d}$ is not equivalent to the Euclidean space $\mathbb{R}^{2 d+1}$, and in fact the distance coincides with the Carnot-Carathéodory distance defined by the sub-Laplacian on $\mathbb{H}_{d}$. The Lie algebra of $\mathbb{H}_{d}$ can be written as the linear span of a family of vector fields $\mathbf{X}=\left\{X_{1}, \ldots, X_{2 d}\right\}$ and of their commutators, which reduce in fact to a single "transversal" vector field $T$. The sub-Laplacian on $\mathbb{H}_{d}$ is the (negative) sum of squares $-\sum_{j=1}^{2 d} X_{j}^{2}$, and thus is of the type studied by Hörmander in [26]. The function spaces that better describe the smoothness of functions in this setting can be defined by their behaviour with respect to the action of only the vector fields $\mathbf{X}$. Such systems of vector fields were called horizontal and they were studied in [13] and [10] and again differed from their Euclidean analogues. In these papers, the authors proved analogue of embedding and interpolation results for the newly defined Sobolev and Lipschitz spaces, in the case of $\mathbb{H}_{d}$, and of Carnot-Carathéodory groups, respectively. ${ }^{1}$

These results gave tremendous impetus to the development of analysis on $\mathbb{H}_{d}$, and more in general on Carnot-Carathéodory groups. In a series of papers, F. Ricci and E. M. Stein [42-44] studied the boundedness of singular integrals on nilpotent Lie groups, exploring again the connection between the geometry of the metric balls, the size properties of the integral kernels, and the boundedness of the singular integral operators. In [48] R. Strichartz pointed out the importance of the role of the joint spectrum of the sub-Laplacian and $T$. In two fundamental papers, [35, 36] D. Müller, F. Ricci, and E. M. Stein then proved the boundedness of joint spectral multipliers of the sub-Laplacian and $T$ on $\mathbb{H}_{d}$ and the closely related Heisenberg type groups results that were effectively extended to more general groups, though in a slightly different way, by A. Martini [32, Theorem 5.7]. Other related results, on spaces of differential forms, in the spirit of this discussion are [37, 38] and [39, 40].

Thus, a common theme of this circle of ideas is that the underlying manifold, Riemannian or sub-Riemannian, and the collection of vector fields $\mathbf{X}$ satisfying Hörmander's condition and defining the corresponding sub-Laplacian determine a metric structure. The most efficient way to describe smoothness of functions and regularity of canonical operators is via a scale of spaces that are modeled by the sub-Laplacian, hence by $\mathbf{X}$, itself.

In the setting of Carnot-Carathéodory groups, and more in general of Lie groups of polynomial growth, endowed with the sub-Riemannian structure induced by a family $\mathbf{X}$ of vector fields satisfying Hörmander's condition, a MihlinHörmander multiplier theorem holds. This fact allowed G. Furioli, C. Melzi and A. Veneruso [14] to introduce Besov spaces on such groups, which were later studied by I. Gallagher and Y. Sire [16]. The theory was recently extended to any unimodular Lie group by J. Feneuil [9].

This work aims to contribute to the analysis of function spaces on general noncompact Lie groups, hence including the nonunimodular groups, with Haar measures of exponential growth.

\footnotetext{
${ }^{1}$ In [10] the Carnot-Carathéodory groups were called stratified nilpotent Lie groups.
} 
Concerning the function spaces, their algebra properties are of great importance, in particular in application to well-posedness results for nonlinear differential equations. In this direction, a remarkable paper is [7] by T. Coulhon, E. Russ and V. Tardivel-Nachev, where they proved algebra properties for the Sobolev spaces, in particular on any unimodular Lie group. The algebra properties were extended to the scale of Besov spaces on groups of polynomial growth in [16] and in [9] on unimodular Lie groups.

A number of the aforementioned results were also obtained in the context of doubling measure metric spaces with the reverse doubling property, see e.g. [22,34], and in the setting of Riemannian manifolds of bounded geometry, see e.g. [4951], [7], and references therein. On the other hand, not much is known in the setting of a sub-Riemannian manifold. This work is part of a program [41], [5] and [6], whose main long term goal is to address this type of questions on a sub-Riemannian manifold, and we started with the case of a general Lie group. The paper [41] studies Sobolev spaces with respect to the sum-of-squares sub-Laplacian, results then extended to Sobolev spaces with respect to sub-Laplacians with drift in [5], while in [6] we develop the theory of Besov and Triebel-Lizorkin spaces with respect to sub-Laplacians with drift, that we further analyse in this work.

We conclude this part of the introduction by pointing out that the literature in this area is extremely vast, and it is just impossible to give credit to all the authors that have contributed to its development. We apologise to everyone whom we did not explicitly mention.

Let $G$ be a noncompact connected Lie group and let $\mathbf{X}=\left\{X_{1}, \ldots, X_{\ell}\right\}$ be a family of linearly independent left-invariant vector fields on $G$ satisfying Hörmander's condition. We denote by $\delta$ the modular function on $G$. Let $\rho$ be a right Haar measure of $G$, let $\chi$ be a continuous positive character of $G$, and consider the measure $\mu_{\chi}$ defined by the relation $d \mu_{\chi}=\chi d \rho$. Consider now the differential operator

$$
\Delta_{\chi}=-\sum_{j=1}^{\ell}\left(X_{j}^{2}+c_{j} X_{j}\right)
$$

with domain $C_{c}^{\infty}(G)$, where $c_{j}=\left(X_{j} \chi\right)(e), j=1, \ldots, \ell$, and $e$ is the identity of G.

This operator was introduced by W. Hebisch et al. in [23], where they showed that $\Delta_{\chi}$ is essentially self-adjoint on $L^{2}\left(\mu_{\chi}\right)$. Moreover, they proved that if a subLaplacian with drift is symmetric on $L^{2}(\mu)$ for a positive measure $\mu$ on $G$, then necessarily $\mu=\mu_{\chi}$ for a positive character $\chi$ on $G$, and moreover the drift has the form $X:=\sum_{j=1}^{\ell} c_{j} X_{j}$, where $c_{j}=\left(X_{j} \chi\right)(e), j=1, \ldots, \ell$, as in (1). Notice that when the character $\chi$ is the modular function, $\mu_{\delta}=\lambda$ is a left Haar measure and the operator $\Delta_{\delta}$ coincides with the intrinsic hypoelliptic Laplacian associated with the Carnot-Carathéodory metric induced on $G$ by the vector fields $\mathbf{X}$ - see [1]. The operator $\Delta_{\delta}$ is the natural substitute of the Laplacian on a general Lie group $G$. This also reflects on the fact that the measure $\lambda$ is privileged among the measures $\mu_{\chi}$. As 
shown in [1,5], $\Delta_{\delta}$ is not a sum-of-squares operator unless the group is unimodular. In this paper we continue the study of function spaces associated with $\Delta_{\chi}$ for a generic positive continuous character $\chi$. The more general treatment allows extra flexibility, see e.g. the embedding results, Theorems 1.1 and 4.4 in [5] and Theorems 5.2 and 5.3 in [6], and at the same time, highlights the naturality of $\Delta_{\delta}$.

In this paper we further develop the investigation of Besov and Triebel-Lizorkin spaces on $G$, defined in terms of $\Delta_{\chi}$, spaces that were introduced by the authors in the recent paper [6].

We prove characterizations of the norms in term of finite differences (Theorems 8 and 9), the density of the test functions in Besov and Triebel-Lizorkin spaces, and the boundedness of a simplified version of the local Riesz transforms (Theorem 13).

The plan of the paper is as follows. In the next section we recall the basic facts about our setting and in particular the heat semigroup generated by $\Delta_{\chi}$. In Sect. 3 we recall the definitions of Besov and Triebel-Lizorkin spaces, and the results of [6] needed in the present work. In Sect. 4 we prove finite difference characterizations for the Besov and Triebel-Lizorkin spaces. Such characterizations are then used in Sect. 5 to show that test functions are dense in such spaces, and in Sect. 6 we prove an isomorphism result and the boundedness of the aforementioned version of the local Riesz transforms for both scales of Besov and Triebel-Lizorkin spaces. We conclude by mentioning some directions for future work.

We shall denote by $C$ a positive constant that may vary from place to place, and may depend on any factor quantified (implicitly or explicitly) before its occurrence, but not on factors quantified afterwards. For any quantities $A$ and $B$, we write $A \lesssim B$ to indicate that there exists a constant $C>0$ as above such that $A \leq C B$. If $A \lesssim B$ and $B \lesssim A$, we write $A \approx B$. In order to emphasize the dependence on a given parameter, say $R$, we write $\lesssim_{R}$, and analogously for the other cases.

Foreword by the Second Named Author Soon after getting my Ph. D., I obtained a position at the Politecnico in Torino, where Fulvio had been for a number of years. He was my main reason for seeking this position at the Politecnico. I immediately found myself immersed in a very pleasant environment, with Fulvio being the organiser of many activities, such as advanced courses, regular seminars, and the visits of many leading mathematicians. I was exposed to a flurry of recent and as well as ongoing research, on a variety of different topics. This gave me the possibility of meeting and interacting with many experts. Fulvio personally introduced me to this world, taking the time to explain to me a lot of mathematics, while advising and guiding me. I have always been very impressed by his poise, kindness, and, most of all, generosity in teaching all the younger mathematicians who had the fortune to interact with him. He has had a great impact on me, both professionally and personally.

I wish to express to Fulvio my most sincere gratitude for all he has taught me, and for his invaluable friendship. 


\section{Basic Facts and Definitions}

Let $G$ be any Lie group with identity element $e$. We denote by $\rho$ a right Haar measure, and by $\delta$ the modular function. We let $\lambda$ be the left Haar measure such that $d \lambda=\delta d \rho$. We recall that $\delta$ is a smooth positive character, that is, a smooth group homomorphism of $G$ onto $\mathbb{R}^{+}$. If $\chi$ is any continuous positive character of $G$, then $\chi$ is automatically smooth. For any such $\chi$, we define $\mu_{\chi}$ to be the measure whose density with respect to $\rho$ is $\chi$, that is, $d \mu_{\chi}=\chi d \rho$. Notice that $\mu_{1}=\rho$ and $\mu_{\delta}=\lambda$.

We fix once for all a family of left-invariant linearly independent vector fields $\mathbf{X}=\left\{X_{1}, \ldots, X_{\ell}\right\}$ satisfying Hörmander's condition. These vector fields induce the Carnot-Charathéodory distance, denoted by $d_{C}$, which turns out to be left-invariant. Then, for $x \in G$ we set $|x|=d_{C}(x, e)$ and we denote with $B(x, r)$ the ball centered at $x$ and of radius $r>0$. If $x=e$ and $r>0$, we write $B_{r}=B(e, r)$, and define $V(r)=\rho\left(B_{r}\right)$. In general, we denote by $B\left(x_{0}, r\right)$ the ball with center $x_{0}$ and radius $r$, in the metric $d_{C}$.

It is known that there exist two constants $d, D>0$ such that

$$
\begin{cases}\rho\left(B_{r}\right) \approx r^{d} & \text { if } r \in(0,1] \\ \rho\left(B_{r}\right) \lesssim e^{D r} & \text { if } r \in(1,+\infty),\end{cases}
$$

see [21, 55]. It is worth pointing out that $d=d(\mathbf{X}, G)$, while $D=D(G)$.

We observe that, having fixed $R>0$, every character $\chi$ satisfies the estimates

$$
\chi(x) \approx_{R} \chi(y)
$$

for all $x, y \in G$ such that $d_{C}(x, y) \leq R$. This equivalence easily implies that $\left(G, d_{C}, \mu_{\chi}\right)$ is locally doubling, that is, for all $0<r<R$ and $x_{0} \in G$,

$$
\mu_{\chi}\left(B\left(x_{0}, 2 r\right) \lesssim_{R} \mu_{\chi}\left(B\left(x_{0}, r\right)\right) .\right.
$$

Having fixed $\mathbf{X}$, we consider the operator $\Delta_{\chi}$ defined in (1). With an abuse of notation, we still denote by $\Delta_{\chi}$ its smallest closed extension on $L^{p}\left(\mu_{\chi}\right)$, where, for $p \in(1,+\infty), L^{p}\left(\mu_{\chi}\right)$ denotes the standard Lebesgue space. The space $L^{\infty}$ is the space of $\rho$-essentially bounded functions. We refer to [23] and [5] for further details about $\Delta_{\chi}$.

We set $\mathbb{J}=\{1, \ldots, \ell\}$ and we say that a multi-index $J=\left(j_{1}, \ldots, j_{m}\right) \in \mathbb{J}^{m}$ if $j_{k} \in \mathbb{J}$ for $k=1, \ldots, m$. Moreover, we write

$$
X_{J}=X_{j_{1}} \cdots X_{j_{m}} .
$$

Next, we observe that, since $\Delta_{\chi}$ is left-invariant, the associated heat semigroup admits a convolution kernel $p_{t}^{\chi} \in \mathcal{D}^{\prime}(G)$, i.e.

$$
e^{-t \Delta_{\chi}} f=f * p_{t}^{\chi} .
$$


It is known (see [5]) that

$$
p_{t}^{\chi}=e^{-c_{X}^{2} t / 4} \chi^{-1 / 2} p_{t}
$$

where $c_{X}=\left(\sum_{j=1}^{\ell}\left(X_{j} \chi(e)\right)^{2}\right)^{1 / 2}$, and $p_{t}$ is the convolution kernel of the heat semigroup in the case $\chi=1$, for which the estimates in [56] are available.

We recall the expressions of the convolution on $G$. We have

$$
\begin{aligned}
f * g(x) & =\int_{G} f\left(x y^{-1}\right) g(y) d \rho(y)=\int_{G} f\left(y^{-1}\right) g(y x) d \rho(y) \\
& =\int_{G} f(y) g\left(y^{-1} x\right) d \lambda(y)=\int_{G} f(x y) g\left(y^{-1}\right) d \lambda(y) .
\end{aligned}
$$

The following result is essentially Lemma 3.1 in [6].

Lemma 1 The following properties hold:

(i) $\left(e^{-t \Delta_{\chi}}\right)_{t>0}$ is a diffusion semigroup on $\left(G, \mu_{\chi}\right)$;

(ii) for every $r>0, \sup _{B_{r}} \chi=e^{c_{X} r}$;

(iii) there exist two constants $c_{1}, c_{2}>0$ such that

$$
\left(\delta \chi^{-1}\right)^{1 / 2}(x) V(\sqrt{t})^{-1} e^{-c_{1}|x|^{2} / t} \lesssim p_{t}^{\chi}(x) \lesssim\left(\delta \chi^{-1}\right)^{1 / 2}(x) V(\sqrt{t})^{-1} e^{-c_{2}|x|^{2} / t}
$$

for every $t \in(0,1)$ and $x \in G$;

(iv) given $m \in \mathbb{N}$, there exist a positive constant $b=b_{m}$ such that

$$
\left|X_{J} p_{t}^{\chi}(x)\right| \lesssim\left(\delta \chi^{-1}\right)^{1 / 2}(x) t^{-m / 2} V(\sqrt{t})^{-1} e^{-b|x|^{2} / t}
$$

for every $x \in G, J \in \mathbb{J}^{m}$ and $t \in(0,1)$;

(v) there exists $c_{3}>0$ such that

$$
\left|\frac{\partial}{\partial t} p_{t}^{\chi}(x)\right| \lesssim\left(\delta \chi^{-1}\right)^{1 / 2}(x) t^{-1} V(\sqrt{t})^{-1} e^{-c_{3}|x|^{2} / t}
$$

for every $t \in(0,1)$ and $x \in G$.

Definition 2 We define the space $\mathcal{S}(G)$ as the space of functions $\varphi \in C^{\infty}(G)$ such that for all $n, m \in \mathbb{N}, J \in \mathbb{J}^{m}$ the seminorms

$$
\mathcal{N}_{J, n}(\varphi)=\sup _{x \in G} e^{n|x|}\left|X_{J} \varphi(x)\right|
$$

are finite. The space $\mathcal{S}^{\prime}(G)$ is defined as the dual space of $\mathcal{S}(G)$. 
In particular, every function $g \in L_{l o c}^{1}(G)$ and of most exponential growth, that is such that $e^{-c|x|} g \in L^{\infty}(G)$ for some $c>0$, will be identified in the sequel with a distribution in $\mathcal{S}^{\prime}(G)$ as

$$
\langle g, \varphi\rangle=\int_{G} g(x) \varphi(x) d \rho(x) \quad \forall \varphi \in \mathcal{S}(G) .
$$

Observe moreover that, if $G$ has polynomial volume growth, then $\mathcal{S}(G)$ is a subset of the usual Schwartz space on $G$. Indeed, if one defines $\mathcal{S}_{\chi}(G)$ as the space of functions $\varphi \in C^{\infty}(G)$ such that for all $n, m \in \mathbb{N}, J \in \mathbb{J}^{m}$ the seminorms

$$
\mathcal{N}_{J, n}^{\chi}(\varphi)=\sup _{x \in G}\left(1+\mu_{\chi}(B(e,|x|))\right)^{n}\left|X_{J} \varphi(x)\right|
$$

are finite, then $\mathcal{S}(G) \subseteq \mathcal{S}_{\chi}(G)$ for any character $\chi$ by Lemma 1 (ii).

For simplicity of notation, we write $\mathcal{S}$ and $\mathcal{S}^{\prime}$ in place of $\mathcal{S}(G)$ and $\mathcal{S}^{\prime}(G)$ respectively. As a consequence of the Gaussian estimates (iv) in Lemma 1, we have the following simple lemma.

Lemma 3 For all $t>0, p_{t}^{\chi} \in \mathcal{S}$. Moreover, $e^{-t \Delta_{\chi}}: \mathcal{S} \rightarrow \mathcal{S}$ is bounded, with seminorms uniformly bounded for $t \in[\varepsilon, R]$, for any $0<\varepsilon<R$. Therefore, $e^{-t \Delta_{\chi}}$ extends to a continuous map $e^{-t \Delta_{\chi}}: \mathcal{S}^{\prime} \rightarrow \mathcal{S}^{\prime}$, for all $t>0$.

Proof We indicate the argument for sake of completeness. Given $n \in \mathbb{N}$ and a multi-index $J$, we have

$$
\begin{aligned}
e^{n|x|}\left|X_{J}\left(\varphi * p_{t}^{\chi}\right)(x)\right| & =e^{n|x|}\left|\left(\varphi * X_{J} p_{t}^{\chi}\right)(x)\right| \\
& \leq \int_{G} e^{n\left|x y^{-1}\right|}\left|\varphi\left(x y^{-1}\right)\right| e^{n|y|}\left|X_{J} p_{t}^{\chi}(y)\right| d \rho(y) \\
& \leq \mathcal{N}_{0, n}(\varphi) \int_{G} e^{n|y|}\left|X_{J} p_{t}^{\chi}(y)\right| d \rho(y) \\
& \lesssim t^{-|J| / 2} \mathcal{N}_{0, n}(\varphi),
\end{aligned}
$$

where the last inequality is obtained arguing as in the proof of Lemma 3.4 in [6]. The conclusions now follow easily.

Definition 4 For $m \in \mathbb{N}$ and $t>0$, we define the operator $W_{t}^{(m)}$ by setting

$$
W_{t}^{(m)}=\left(t \Delta_{\chi}\right)^{m} e^{-t \Delta_{\chi}}
$$

For $m \in \mathbb{N}$ and $t>0, W_{t}^{(m)}: \mathcal{S} \rightarrow \mathcal{S}$ is bounded, and therefore it extends to a continuous map $W_{t}^{(m)}: \mathcal{S}^{\prime} \rightarrow \mathcal{S}^{\prime}$. We also observe that, for $f \in \mathcal{S}^{\prime}, W_{t}^{(m)} f$ is a $C^{\infty}$ function, for all $t>0$ and $m \in \mathbb{N}$. 
We also recall the definition of the Littlewood-Paley-Stein $g$-function. Given a positive integer $k$, for $f \in \mathcal{S}$ we set

$$
g_{k}(f)=\left(\int_{0}^{+\infty}\left|W_{s}^{(k)} f\right|^{2} \frac{d s}{s}\right)^{1 / 2} .
$$

Since $\Delta_{\chi}$ generates a symmetric diffusion semigroup, if $p \in(1,+\infty)$ then $g_{k}$ satisfies the estimate

$$
\left\|g_{k}(f)\right\|_{L^{p}\left(\mu_{\chi}\right)} \approx\|f\|_{L^{p}\left(\mu_{\chi}\right)}
$$

for any $f \in L^{p}\left(\mu_{\chi}\right)$, see [47], and also [33].

\section{Triebel-Lizorkin and Besov Spaces on $G$}

Here and in what follows, given a measure space $(\Omega, v)$ and a Banach space $\mathcal{X}$, for $p \in[1,+\infty]$, we denote by $L^{p}(\Omega, v ; \mathcal{X})$ the space of measurable functions $f: \Omega \rightarrow \mathcal{X}$ such that

$$
\|f\|_{L^{p}(\Omega, v ; \mathcal{X})}:=\left\{\int_{\Omega}\|f(\omega)\|_{\mathcal{X}}^{p} d \nu(\omega)\right\}^{1 / p}<\infty
$$

when $p \in[1,+\infty)$, with the obvious modification if $p=+\infty$. We also denote by $[\tau]$ the integral part of $\tau \geq 0$.

We are now in the position to introduce the Triebel-Lizorkin and Besov spaces on $G$, defined in terms of the sub-Laplacian $\Delta_{\chi}$, see [6].

Definition 5 Let $p, q \in[1,+\infty]$, and $\alpha \geq 0$. Then we define:

(1) the Triebel-Lizorkin space $F_{\alpha}^{p, q}\left(\mu_{\chi}\right)$ as

$$
\begin{array}{r}
F_{\alpha}^{p, q}\left(\mu_{\chi}\right)=\left\{f \in \mathcal{S}^{\prime}(G): t^{-\alpha / 2} W_{t}^{([\alpha / 2]+1)} f \in L^{p}\left(G, \mu_{\chi} ; L^{q}((0,1), d t / t)\right)\right. \\
\text { and } \left.e^{-\frac{1}{2} \Delta_{\chi}} f \in L^{p}\left(\mu_{\chi}\right)\right\}
\end{array}
$$

endowed with the norm

$$
\|f\|_{F_{\alpha}^{p, q}}:=\mathbb{F}_{\alpha}^{p, q}(f)+\left\|e^{-\frac{1}{2} \Delta_{\chi}} f\right\|_{L^{p}\left(\mu_{\chi}\right)},
$$

where

$$
\mathbb{F}_{\alpha}^{p, q}(f):=\left\|\left(\int_{0}^{1}\left(t^{-\alpha / 2}\left|W_{t}^{([\alpha / 2]+1)} f\right|\right)^{q} \frac{d t}{t}\right)^{1 / q}\right\|_{L^{p}\left(\mu_{\chi}\right)}
$$


if $q<+\infty$, while

$$
\mathbb{F}_{\alpha}^{p, \infty}(f):=\left\|\sup _{t \in(0,1)} t^{-\alpha / 2}\left|W_{t}^{([\alpha / 2]+1)} f\right|\right\|_{L^{p}\left(\mu_{\chi}\right)}
$$

(2) the Besov space $B_{\alpha}^{p, q}\left(\mu_{\chi}\right)$ as

$$
\begin{array}{r}
B_{\alpha}^{p, q}\left(\mu_{\chi}\right)=\left\{f \in \mathcal{S}^{\prime}(G): t^{-\alpha / 2} W_{t}^{([\alpha / 2]+1)} f \in L^{q}\left((0,1), d t / t ; L^{p}\left(G, \mu_{\chi}\right)\right)\right. \\
\text { and } \left.e^{-\frac{1}{2} \Delta_{\chi}} f \in L^{p}\left(\mu_{\chi}\right)\right\}
\end{array}
$$

endowed with the norm

$$
\|f\|_{B_{\alpha}^{p, q}}:=\mathbb{B}_{\alpha}^{p, q}(f)+\left\|e^{-\frac{1}{2} \Delta_{\chi}} f\right\|_{L^{p}\left(\mu_{\chi}\right)},
$$

where

$$
\mathbb{B}_{\alpha}^{p, q}(f):=\left(\int_{0}^{1}\left(t^{-\alpha / 2}\left\|W_{t}^{([\alpha / 2]+1)} f\right\|_{L^{p}\left(\mu_{\chi}\right)}\right)^{q} \frac{d t}{t}\right)^{1 / q}
$$

if $q<+\infty$, while

$$
\mathbb{B}_{\alpha}^{p, \infty}(f):=\sup _{t \in(0,1)} t^{-\alpha / 2}\left\|W_{t}^{([\alpha / 2]+1)} f\right\|_{L^{p}\left(\mu_{\chi}\right)} .
$$

We emphasize that, when $p \in(1,+\infty)$ and $\alpha \geq 0$, the Triebel-Lizorkin space $F_{\alpha}^{p, 2}\left(\mu_{\chi}\right)$ coincides with the Sobolev space $L_{\alpha}^{p}\left(\mu_{\chi}\right)$ defined in [5], with equivalence of norms, see [6, Theorem 5.2].

We now recall the main results in [6] about equivalence of norms in Besov and Triebel-Lizorkin spaces. The following is [6, Theorem 4.1].

Theorem 6 Let $\alpha>0, m>\alpha / 2$ be an integer, $t_{0} \in[0,1)$ and $q \in[1,+\infty]$.

(i) If $p \in(1,+\infty)$, then the norm $\|f\|_{F_{\alpha}^{p, q}}$ is equivalent to the norm

$$
\left\|\left(\int_{0}^{1}\left(t^{-\alpha / 2}\left|W_{t}^{(m)} f\right|\right)^{q} \frac{d t}{t}\right)^{1 / q}\right\|_{L^{p}\left(\mu_{\chi}\right)}+\left\|e^{-t_{0} \Delta_{\chi}} f\right\|_{L^{p}\left(\mu_{\chi}\right)} .
$$

(ii) If $p \in[1,+\infty]$, then the norm $\|f\|_{B_{\alpha}^{p, q}}$ is equivalent to the norm

$$
\left(\int_{0}^{1}\left(t^{-\alpha / 2}\left\|W_{t}^{(m)} f\right\|_{L^{p}\left(\mu_{\chi}\right)}\right)^{q} \frac{d t}{t}\right)^{1 / q}+\left\|e^{-t_{0} \Delta_{\chi}} f\right\|_{L^{p}\left(\mu_{\chi}\right)} .
$$


If $\alpha=0$, the norms $\|f\|_{F_{\alpha}^{p, q}}$ and $\|f\|_{B_{\alpha}^{p, q}}$ are equivalent to those in (12) and (13) respectively provided $t_{0} \in(0,1)$.

The next result concerns a discretization of the norm that resembles the Littlewood-Paley characterization of Besov and Triebel-Lizorkin spaces in the classical cases. In our case, for $j \in \mathbb{N}$, the operators $W_{2^{-j}}^{(m)}$ play the role of the operators $\triangle_{j}$ in the classical Littlewood-Paley decomposition, while $e^{-t_{0} \Delta_{\chi}}$ plays the role of $S_{0}$; see e.g. [20] for such notation in the case of $\mathbb{R}^{d}$.

We point out that in the case of $\Delta_{\chi}$ the classical Littlewood-Paley characterization of Besov and Triebel-Lizorkin spaces cannot hold since any bounded spectral multiplier of $\Delta_{\chi}$ on $L^{p}\left(\mu_{\chi}\right)$, with $p \neq 2$, admits a holomorphic extension to a parabolic region in $\mathbb{C}$, see [23]. This is [6, Theorem 4.2].

Theorem 7 Let $\alpha>0, m>\alpha / 2$ be an integer, $t_{0} \in[0,1)$ and $q \in[1,+\infty]$.

(i) If $p \in(1,+\infty)$, then the norm $\|f\|_{F_{\alpha}^{p, q}}$ is equivalent to the norm

$$
\left\|\left(\sum_{j=0}^{\infty}\left(2^{j \alpha / 2}\left|W_{2^{-j}}^{(m)} f\right|\right)^{q}\right)^{1 / q}\right\|_{L^{p}\left(\mu_{\chi}\right)}+\left\|e^{-t_{0} \Delta_{\chi}} f\right\|_{L^{p}\left(\mu_{\chi}\right)} .
$$

(ii) If $p \in[1,+\infty]$, then the norm $\|f\|_{B_{\alpha}^{p, q}}$ is equivalent to the norm

$$
\left(\sum_{j=0}^{\infty}\left(2^{j \alpha / 2}\left\|W_{2^{-j}}^{(m)} f\right\|_{L^{p}\left(\mu_{\chi}\right)}\right)^{q}\right)^{1 / q}+\left\|e^{-t_{0} \Delta_{\chi}} f\right\|_{L^{p}\left(\mu_{\chi}\right)} .
$$

If $\alpha=0$, the norms $\|f\|_{F_{\alpha}^{p, q}}$ and $\|f\|_{B_{\alpha}^{p, q}}$ are equivalent respectively to those in (14) and (15) provided $t_{0} \in(0,1)$.

\section{Finite Differences Characterizations}

In this section we prove characterizations for the spaces $F_{\alpha}^{p, q}$ and $B_{\alpha}^{p \cdot q}$ in terms of finite differences. Such characterizations provide a key tool for the proof of the density lemma of Sect. 5 . We begin by introducing the finite difference operator.

Given a measurable function $f$, for $x, y \in G$ we define

$$
\mathrm{D}_{y} f(x)=f\left(x y^{-1}\right)-f(x) \text {. }
$$




\subsection{Characterization of Triebel-Lizorkin Norm by Differences}

For $q \in[1, \infty]$ and $\alpha \in(0,1)$, we define the functional

$$
\mathbb{S}_{\alpha}^{\text {loc, } q} f(x)=\left(\int_{0}^{1}\left[\frac{1}{u^{\alpha} V(u)} \int_{|y|<u}\left|\mathrm{D}_{y} f(x)\right| d \rho(y)\right]^{q} \frac{d u}{u}\right)^{1 / q} .
$$

We point out that in the case $q=2$, such functional coincides with the classical functional $\mathbb{S}_{\alpha}^{\text {loc }}$ used to characterize the Sobolev norm, see [7] for the unimodular case, and [5] for the nonunimodular (and weighted) case.

The first result of this section is the characterization of the Triebel-Lizorkin norm of $F_{\alpha}^{p, q}$ in terms of the $L^{p}\left(\mu_{\chi}\right)$-integrability of the functional $\mathbb{S}_{\alpha}^{\text {loc, } q}$.

Theorem 8 For every $p, q \in(1, \infty), \alpha \in(0,1)$, we have

$$
\|f\|_{F_{\alpha}^{p, q}} \approx\left\|\mathbb{S}_{\alpha}^{\text {loc, } q} f\right\|_{L^{p}\left(\mu_{\chi}\right)}+\|f\|_{L^{p}\left(\mu_{\chi}\right)}
$$

for any $f \in F_{\alpha}^{p, q}\left(\mu_{\chi}\right)$.

\section{Proof Set}

$$
H_{\alpha, q} f:=\left(\int_{0}^{1}\left(t^{-\alpha / 2}\left|W_{t}^{(1)} f\right|\right)^{q} \frac{d t}{t}\right)^{1 / q},
$$

and observe that, since $\alpha \in(0,1)$,

$$
\|f\|_{F_{\alpha}^{p, q}} \lesssim\left\|H_{\alpha, q} f\right\|_{L^{p}\left(\mu_{\chi}\right)}+\|f\|_{L^{p}\left(\mu_{\chi}\right)} .
$$

Step 1 We shall prove that, for all $f \in F_{\alpha}^{p, q}$,

$$
\|f\|_{F_{\alpha}^{p, q}} \lesssim\left\|\mathbb{S}_{\alpha}^{\mathrm{loc}, q} f\right\|_{L^{p}\left(\mu_{\chi}\right)}+\|f\|_{L^{p}\left(\mu_{\chi}\right)}
$$

by showing that

$$
\left\|H_{\alpha, q} f\right\|_{L^{p}\left(\mu_{\chi}\right)} \lesssim\left\|\mathbb{S}_{\alpha}^{\operatorname{loc}, q} f\right\|_{L^{p}\left(\mu_{\chi}\right)}+\|f\|_{L^{p}\left(\mu_{\chi}\right)} .
$$

We first notice that for every $t \in(0,1)$ and $x \in G$, since $\frac{\partial}{\partial t} \int_{G} p_{t}^{\chi} d \rho=0$, we have

$$
\begin{aligned}
\left|\Delta_{\chi} e^{-t \Delta_{\chi}} f(x)\right| & =\left|\frac{\partial}{\partial t} e^{-t \Delta_{\chi}} f(x)\right| \\
& =\left|\frac{\partial}{\partial t}\left(\int_{G} f\left(x y^{-1}\right) p_{t}^{\chi}(y) d \rho(y)-\int_{G} f(x) p_{t}^{\chi}(y) d \rho(y)\right)\right| \\
& \leq \int_{G}\left|\mathrm{D}_{y} f(x)\right|\left|\frac{\partial p_{t}^{\chi}(y)}{\partial t}\right| d \rho(y) .
\end{aligned}
$$


Using the estimates (v) of Lemma 1 we have

$$
\begin{aligned}
& \left(H_{\alpha, q} f(x)\right)^{q} \\
& \lesssim \int_{0}^{1} t^{-q \alpha / 2} V(\sqrt{t})^{-q}\left(\int_{|y|<\sqrt{t}}\left|\mathrm{D}_{y} f(x)\right|\left(\delta \chi^{-1}\right)^{1 / 2}(y) e^{-c_{3}|y|^{2} / t} d \rho(y)\right)^{q} \frac{d t}{t} \\
& \quad+\sum_{k=0}^{\infty} \int_{0}^{1} t^{-q \alpha / 2} V(\sqrt{t})^{-q} \\
& \quad \times\left(\int_{2^{k} \sqrt{t}<|y|<2^{k+1} \sqrt{t}}\left|\mathrm{D}_{y} f(x)\right|\left(\delta \chi^{-1}\right)^{1 / 2}(y) e^{-c_{3}|y|^{2} / t} d \rho(y)\right)^{q} \frac{d t}{t} \\
& \lesssim \int_{0}^{1} t^{-q \alpha / 2} V(\sqrt{t})^{-q}\left(\int_{|y|<\sqrt{t}}\left|\mathrm{D}_{y} f(x)\right| d \rho(y)\right)^{q} \frac{d t}{t} \\
& \quad+\sum_{k=0}^{\infty} e^{-c_{3} 2^{2 k}} \int_{0}^{1} t^{-q \alpha / 2} V(\sqrt{t})^{-q} \\
& \quad \times\left(\int_{|y|<2^{k+1} \sqrt{t}}^{\left.\left|\mathrm{D}_{y} f(x)\right|\left(\delta \chi^{-1}\right)^{1 / 2}(y) d \rho(y)\right)^{q} \frac{d t}{t} .}\right.
\end{aligned}
$$

By the change of variables $u=2^{k+1} \sqrt{t}$ we obtain

$$
\begin{aligned}
& \left(H_{\alpha, q} f(x)\right)^{q} \\
& \lesssim \int_{0}^{1} \frac{1}{u^{q \alpha} V(u)^{q}}\left(\int_{|y|<u}\left|\mathrm{D}_{y} f(x)\right| d \rho(y)\right)^{q} \frac{d u}{u} \\
& +\sum_{k=0}^{\infty} e^{-c_{3} 2^{2 k}} \int_{0}^{2^{k+1}} \frac{2^{(k+1) q \alpha}}{u^{q \alpha} V\left(2^{-k-1} u\right)^{q}} \\
& \quad \times\left(\int_{|y|<u}\left|\mathrm{D}_{y} f(x)\right|\left(\delta \chi^{-1}\right)^{1 / 2}(y) d \rho(y)\right)^{q} \frac{d u}{u} \\
& \lesssim\left(\mathbb{S}_{\alpha}{ }^{\circ o c, q} f(x)\right)^{q} \\
& +\sum_{k=0}^{\infty} e^{-c_{3} 2^{2 k}} \int_{0}^{1} \frac{2^{(k+1) q \alpha}}{u^{q \alpha} V\left(2^{-k-1} u\right)^{q}}\left(\int_{|y|<u}\left|\mathrm{D}_{y} f(x)\right| d \rho(y)\right)^{q} \frac{d u}{u} \\
& +\sum_{k=0}^{\infty} e^{-c_{3} 2^{2 k}} \int_{1}^{2^{k+1}} \frac{2^{(k+1) q \alpha}}{u^{q \alpha} V\left(2^{-k-1} u\right)^{q}} \\
& \quad \times\left(\int_{|y|<u}\left|\mathrm{D}_{y} f(x)\right|\left(\delta \chi^{-1}\right)^{1 / 2}(y) d \rho(y)\right)^{q} \frac{d u}{u} .
\end{aligned}
$$


By the estimates (2), we obtain that

$$
\begin{aligned}
& \left(H_{\alpha, q} f(x)\right)^{q} \\
& \lesssim\left(\mathbb{S}_{\alpha}^{\operatorname{loc}, q} f(x)\right)^{q}+\left(\mathbb{S}_{\alpha}^{\operatorname{loc}, q} f(x)\right)^{q} \sum_{k=0}^{\infty} e^{-c_{3} 2^{2 k}} 2^{(k+1)(q \alpha+q d)} \\
& +\sum_{k=0}^{\infty} e^{-c_{3} 2^{2 k}} 2^{(k+1)(q \alpha+q d)} \int_{1}^{2^{k+1}} \frac{1}{u^{q \alpha+q d}}|f(x)|^{q} \\
& \quad \times\left(\int_{|y|<u}\left(\delta \chi^{-1}\right)^{1 / 2}(y) d \rho(y)\right)^{q} \frac{d u}{u} \\
& +\sum_{k=0}^{\infty} e^{-c_{3} 2^{2 k}} 2^{(k+1)(q \alpha+q d)} \int_{1}^{2^{k+1}} \frac{1}{u^{q \alpha+q d}} \\
& \quad \times\left(\int_{|y|<u}\left|f\left(x y^{-1}\right)\right|\left(\delta \chi^{-1}\right)^{1 / 2}(y) d \rho(y)\right)^{q} \frac{d u}{u} \\
& \lesssim\left(\mathbb{S}_{\alpha}{ }^{(o c, q} f(x)\right)^{q}+\sum_{k=0}^{\infty} J_{k}(x)+\sum_{k=0}^{\infty} I_{k}(x) .
\end{aligned}
$$

By the growth estimates of characters in Lemma 1 (ii) and by (2) we deduce that there exists $C>0$ such that

$$
\begin{aligned}
\sum_{k=0}^{\infty} J_{k}(x) & \lesssim \sum_{k=0}^{\infty} e^{-c_{3} 2^{2 k}} 2^{(k+1)(q \alpha+q d)} \int_{1}^{2^{k+1}} \frac{1}{u^{q \alpha+q d}}|f(x)|^{q} e^{q C u} \frac{d u}{u} \\
& \lesssim \sum_{k=0}^{\infty}|f(x)|^{q} e^{-c_{3} 2^{2 k}} 2^{(k+1)(q \alpha+q d)} e^{q C 2^{k+1}} \\
& \lesssim|f(x)|^{q} .
\end{aligned}
$$

We now notice that there exists $c>0$ such that

$$
\begin{aligned}
& \left\|\left(\sum_{k=0}^{\infty} I_{k}\right)^{1 / q}\right\| L_{L^{p}\left(\mu_{\chi}\right)} \\
& \lesssim \sum_{k=0}^{\infty} e^{-c 2^{2 k}}\left\|\left(\int_{1}^{2^{k+1}}\left(\int_{|y|<u}\left|f\left(\cdot y^{-1}\right)\right|\left(\delta \chi^{-1}\right)^{1 / 2}(y) d \rho(y)\right)^{q}\right)^{1 / q} d u\right\|_{L^{p}\left(\mu_{\chi}\right)} .
\end{aligned}
$$


Here and in the rest of this work, we denote by $\mathbf{1}_{E}$ the characteristic function of the measurable set $E$. For every integer $k$, by Minkowski's integral inequality, we get

$$
\begin{aligned}
& \left(\int_{1}^{2^{k+1}}\left(\int_{G}\left|f\left(x y^{-1}\right)\right| \mathbf{1}_{B_{u}}(y)\left(\delta \chi^{-1}\right)^{1 / 2}(y) d \rho(y)\right)^{q} d u\right)^{1 / q} \\
& \lesssim \int_{G}\left(\int_{1}^{2^{k+1}}\left|f\left(x y^{-1}\right)\right|^{q} \mathbf{1}_{B_{u}}(y)\left(\delta \chi^{-1}\right)^{q / 2}(y) d u\right)^{1 / q} d \rho(y) \\
& \lesssim \int_{B_{1}}\left|f\left(x y^{-1}\right)\right|\left(\delta \chi^{-1}\right)^{1 / 2}(y)\left(\int_{1}^{2^{k+1}} d u\right)^{1 / q} d \rho(y) \\
& \quad+\int_{1<|y|<2^{k+1}}\left|f\left(x y^{-1}\right)\right|\left(\delta \chi^{-1}\right)^{1 / 2}(y)\left(\int_{|y|}^{2^{k+1}} d u\right)^{1 / q} d \rho(y) \\
& \lesssim 2^{k / q} \int_{B_{1}}|f(x z)| d \lambda(z)+2^{k / q} \int_{1<|z|<2^{k+1}}|f(x z)|\left(\delta^{-1} \chi\right)^{1 / 2}(z) d \lambda(z)
\end{aligned}
$$

By applying again Minkowski’s inequality, we then obtain that

$$
\begin{aligned}
& \left\|\left(\int_{1}^{2^{k+1}}\left(\int_{G}\left|f\left(\cdot y^{-1}\right)\right| \mathbf{1}_{B_{u}}(y)\left(\delta \chi^{-1}\right)^{1 / 2}(y) d \rho(y)\right)^{q} d u\right)^{1 / q}\right\|_{L^{p}\left(\mu_{\chi}\right)} \\
& \lesssim 2^{k / q} \int_{B_{1}}\left(\int_{G}|f(x z)|^{p} d \mu_{\chi}(x)\right)^{1 / p} d \lambda(z) \\
& \quad+2^{k / q} \int_{1<|z|<2^{k+1}}\left(\int_{G}|f(x z)|^{p} d \mu_{\chi}(x)\right)^{1 / p}\left(\delta^{-1} \chi\right)^{1 / 2}(z) d \lambda(z) \\
& \lesssim 2^{k / q}\|f\|_{L^{p}\left(\mu_{\chi}\right)}+2^{k / q}\|f\|_{L^{p}\left(\mu_{\chi}\right)} \int_{B_{2^{k+1}}} \chi^{1 / p}\left(\delta \chi^{-1}\right)^{1 / 2} d \rho \\
& \lesssim 2^{k / q} e^{C 2^{k}}\|f\|_{L^{p}\left(\mu_{\chi}\right)} .
\end{aligned}
$$

We then have

$$
\left\|\left(\sum_{k=0}^{\infty} I_{k}\right)^{1 / q}\right\|_{L^{p}\left(\mu_{\chi}\right)} \lesssim \sum_{k=0}^{\infty} e^{-c 2^{2 k}} 2^{k / q+k d} e^{C 2^{k}}\|f\|_{L^{p}\left(\mu_{\chi}\right)}\|f\|_{L^{p}\left(\mu_{\chi}\right)} .
$$

In conclusion, by (21) and (22) we get (19), as required.

It remains to show that for all $f \in F_{\alpha}^{p, q}$

$$
\left\|\mathbb{S}_{\alpha}^{\text {loc, } q} f\right\|_{L^{p}\left(\mu_{\chi}\right)} \lesssim\|f\|_{F_{\alpha}^{p, q} .}
$$


In order to prove this, we write $f=\left(f-e^{-\Delta_{\chi}} f\right)+e^{-\Delta_{\chi}} f$ and we estimate $\left\|\mathbb{S}_{\alpha}^{\mathrm{loc}, q}\left(f-e^{-\Delta_{\chi}} f\right)\right\|_{L^{p}\left(\mu_{\chi}\right)}$ and $\left\|\mathbb{S}_{\alpha}^{\operatorname{loc}, q} e^{-\Delta_{\chi}} f\right\|_{L^{p}\left(\mu_{\chi}\right)}$ separately.

Step 2 We prove that

$$
\left\|\mathbb{S}_{\alpha}^{\operatorname{loc}, q}\left(f-e^{-\Delta_{\chi}} f\right)\right\|_{L^{p}\left(\mu_{\chi}\right)} \lesssim\|f\|_{F_{\alpha}^{p, q}} .
$$

Arguing similarly to [7, 2.1.2] we write

$$
f-e^{-\Delta_{\chi}} f=-\sum_{m=1}^{+\infty} \int_{2^{-m}}^{2^{-m+1}} \frac{\partial}{\partial t} e^{-t \Delta_{\chi}} f d t=: \sum_{m=1}^{+\infty} f_{m}
$$

We then obtain

$$
\begin{aligned}
\left(\mathbb{S}_{\alpha}^{\operatorname{loc}, q}\left(f-e^{-\Delta_{\chi}} f\right)(x)\right)^{q} \\
\left.=\int_{0}^{1}\left(\frac{1}{u^{\alpha} V(u)} \int_{|y|<u} \mid \mathrm{D}_{y}\left(f-e^{-\Delta_{\chi}} f\right)\right)(x) \mid d \rho(y)\right)^{q} \frac{d u}{u} \\
=\sum_{j=1}^{+\infty} \int_{2^{-j}}^{2^{-j+1}}\left(\frac{1}{u^{\alpha} V(u)} \int_{|y|<u}\left|\mathrm{D}_{y}\left(f-e^{-\Delta_{x}} f\right)(x)\right| d \rho(y)\right)^{q} \frac{d u}{u} \\
\lesssim \sum_{j=1}^{+\infty} 2^{j q \alpha}\left(2^{j d} \int_{|y|<2^{-j+1}}\left|\mathrm{D}_{y}\left(f-e^{-\Delta_{x}} f\right)(x)\right| d \rho(y)\right)^{q} \\
\lesssim \sum_{j=1}^{+\infty} 2^{j q \alpha}\left(\sum_{m=1}^{+\infty} 2^{j d} \int_{|y|<2^{-j+1}}\left|\mathrm{D}_{y} f_{m}(x)\right| d \rho(y)\right)^{q} \\
=\sum_{j=1}^{+\infty} 2^{j q \alpha}\left(2^{j d}\left(\sum_{m=1}^{2 j}+\sum_{m=2 j+1}^{+\infty}\right) \int_{|y|<2^{-j+1}}\left|\mathrm{D}_{y} f_{m}(x)\right| d \rho(y)\right)^{q},
\end{aligned}
$$

where $f_{m}$ is defined in (25). If $m>2 j$, then

$$
2^{j d} \int_{|y|<2^{-j+1}}\left|\mathrm{D}_{y} f_{m}(x)\right| d \rho(y) \lesssim M g_{m+1}(x),
$$

where

$$
g_{m+1}=\int_{2^{-m}}^{2^{-m+1}}\left|\frac{\partial}{\partial t} e^{-t \Delta_{\chi}} f\right| d t
$$


and $M$ is the local maximal function with respect to the right Haar measure,

$$
M f(x)=\sup _{x \in B, r_{B} \leq 1} \frac{1}{\rho(B)} \int_{B}|f| d \rho,
$$

which is bounded on $L^{p}\left(\mu_{\chi}\right)$ for every $p \in(1, \infty)$, see [5, Subsection 5.1].

In order to treat the case when $m \leq 2 j$, we notice that for every $j \geq 1, y \in$ $B_{2^{-j-1}}$ and $x \in G$

$$
\left|\mathrm{D}_{y} f_{m}(x)\right| \leq 2^{-j+1} \sup \left\{\left|X_{i} f_{m}(w)\right|: i=1, \ldots, \ell,\left|w^{-1} x\right| \leq 2^{-j+1}\right\} .
$$

Since

$$
f_{m}=\int_{2^{-m-1}}^{2^{-m}} \frac{\partial}{\partial t}\left(e^{-2 t \Delta_{\chi}} f\right) d t=2 \int_{2^{-m-1}}^{2^{-m}} e^{-t \Delta_{\chi}} \frac{\partial}{\partial t}\left(e^{-t \Delta_{\chi}} f\right) d t,
$$

by applying the estimates of the heat kernel in Lemma 1 (v) and (iii), for every $w$ such that $\left|w^{-1} x\right| \leq 2^{-j+1}$ we have

$$
\begin{aligned}
& \left|X_{i} f_{m}(w)\right| \\
& \lesssim \int_{2^{-m-1}}^{2^{-m}} \int_{G}\left|\frac{\partial}{\partial t}\left(e^{-t \Delta_{\chi}} f\right)(z)\right|\left|X_{i} p_{t}^{\chi}\left(z^{-1} w\right)\right| d \lambda(z) d t \\
& \lesssim \int_{G} \int_{2^{-m-1}}^{2^{-m}}\left|\frac{\partial}{\partial t}\left(e^{-t \Delta_{\chi}} f\right)(z)\right| t^{-1 / 2} V(\sqrt{t})^{-1}\left(\chi^{-1} \delta\right)^{1 / 2}\left(z^{-1} w\right) e^{-b\left|z^{-1} w\right|^{2} / t} d t d \lambda(z) \\
& \lesssim 2^{m / 2} 2^{m d / 2} \int_{G} g_{m}(z)\left(\delta \chi^{-1}\right)^{1 / 2}\left(z^{-1} x\right) e^{-\frac{b}{2} 2^{m}\left|z^{-1} x\right|^{2}} d \lambda(z) \\
& \lesssim 2^{m / 2} e^{-c 2^{-m} \Delta_{\chi}} g_{m}(x)
\end{aligned}
$$

for a suitable constant $c$. From (29) it follows that

$$
2^{j d} \int_{|y|<2^{-j+1}}\left|\mathrm{D}_{y} f_{m}(x)\right| d \rho(y) \lesssim 2^{-j+m / 2} e^{-c 2^{-m} \Delta_{\chi}} g_{m}(x) .
$$

Thus, putting together (26), (27) and (31) we obtain

$$
\begin{aligned}
& \left(\mathbb{S}_{\alpha}^{\text {loc }, q}\left(f-e^{-\Delta_{\chi}} f\right)(x)\right)^{q} \\
& \quad \lesssim \sum_{j=1}^{+\infty} 2^{j q \alpha}\left(\sum_{m=1}^{2 j} 2^{-j+m / 2} e^{-c 2^{-m} \Delta_{\chi}} g_{m}(x)+\sum_{m=2 j+1}^{+\infty} M g_{m+1}(x)\right)^{q} \\
& \quad \lesssim \sum_{j=1}^{+\infty} 2^{j q \alpha}\left(\sum_{m=1}^{2 j} 2^{-j+m / 2} e^{-c 2^{-m} \Delta_{\chi}} g_{m}(x)\right)^{q}+\sum_{j=1}^{+\infty} 2^{j q \alpha}\left(\sum_{m=2 j+1}^{+\infty} M g_{m+1}(x)\right)^{q} \\
& \quad=: \Sigma_{1}(x)+\Sigma_{2}(x) .
\end{aligned}
$$


Now we apply Hölder's inequality to see that, for any $\varepsilon>0$,

$$
\begin{aligned}
& \left(\sum_{m=1}^{2 j} 2^{-j+m / 2} e^{-c 2^{-m} \Delta_{\chi}} g_{m}(x)\right)^{q} \\
& \leq\left(\sum_{m=1}^{2 j} 2^{\varepsilon m q^{\prime}}\right)^{q / q^{\prime}} \sum_{m=1}^{2 j} 2^{-j q+m q / 2-\varepsilon m q}\left(e^{-c 2^{-m} \Delta_{\chi}} g_{m}(x)\right)^{q} \\
& \lesssim \sum_{m=1}^{2 j} 2^{2 j \varepsilon q-j q+m q / 2-\varepsilon m q}\left(e^{-c 2^{-m} \Delta_{\chi}} g_{m}(x)\right)^{q}
\end{aligned}
$$

Therefore, since $\alpha \in(0,1)$, choosing $\varepsilon \in(0,(1-\alpha) / 2)$ we obtain

$$
\begin{aligned}
\Sigma_{1}(x) \lesssim \sum_{m=1}^{+\infty} \sum_{j \geq m / 2} 2^{-j(1-\alpha-2 \varepsilon) q+m q / 2-\varepsilon m q}\left(e^{-c 2^{-m} \Delta_{\chi}} g_{m}(x)\right)^{q} \\
\\
\lesssim \sum_{m=1}^{+\infty}\left(2^{m \alpha / 2} e^{-c 2^{-m} \Delta_{\chi}} g_{m}(x)\right)^{q} .
\end{aligned}
$$

Analogously, using Hölder's inequality again, we see that, for $\varepsilon>0$

$$
\begin{aligned}
\left(\sum_{m=2 j+1}^{+\infty} M g_{m+1}(x)\right)^{q} & \lesssim\left(\sum_{m=2 j+1}^{+\infty} 2^{-\varepsilon m q^{\prime}}\right)^{q / q^{\prime}} \sum_{m=2 j+1}^{+\infty}\left(2^{\varepsilon m} M g_{m+1}(x)\right)^{q} \\
& \lesssim 2^{-2 \varepsilon j q} \sum_{m=2 j+1}^{+\infty}\left(2^{\varepsilon m} M g_{m+1}(x)\right)^{q}
\end{aligned}
$$

so that, if $\varepsilon<\alpha / 2$

$$
\Sigma_{2}(x) \lesssim \sum_{m=1}^{+\infty} \sum_{j \leq m / 2} 2^{\varepsilon m q+j q(\alpha-2 \varepsilon)}\left(M g_{m+1}(x)\right)^{q} \lesssim \sum_{m=1}^{+\infty}\left(2^{m \alpha / 2} M g_{m+1}(x)\right)^{q}
$$

Now, from (32) we have

$$
\left\|\mathbb{S}_{\alpha}^{\operatorname{loc}, q}\left(f-e^{-\Delta_{\chi}} f\right)\right\|_{L^{p}\left(\mu_{\chi}\right)} \lesssim\left\|\Sigma_{1}^{1 / q}\right\|_{L^{p}\left(\mu_{\chi}\right)}+\left\|\Sigma_{2}^{1 / q}\right\|_{L^{p}\left(\mu_{\chi}\right)} .
$$


We first estimate the latter term. By the Fefferman-Stein vector-valued theorem (see [17, p. 481]) with the $L^{p}$-boundedness of the local maximal function, and Hölder's inequality, we have

$$
\begin{aligned}
\left\|\Sigma_{2}^{1 / q}\right\|_{L^{p}\left(\mu_{\chi}\right)} & =\left\|\left(\sum_{m=1}^{+\infty}\left(2^{m \alpha / 2} M g_{m+1}\right)^{q}\right)^{1 / q}\right\|_{L^{p}\left(\mu_{\chi}\right)} \\
& \lesssim\left\|\left(\sum_{m=1}^{+\infty}\left(2^{m \alpha / 2} g_{m+1}\right)^{q}\right)^{1 / q}\right\|_{L^{p}\left(\mu_{\chi}\right)} \\
& \lesssim\left\|\left(\sum_{m=1}^{+\infty} 2^{m \alpha q / 2-m(q-1)} \int_{2^{-m}}^{2^{-m+1}}\left|\frac{\partial}{\partial t} e^{-t \Delta_{\chi}} f\right|^{q} d t\right)^{1 / q}\right\|_{L^{p}\left(\mu_{\chi}\right)} \\
& \lesssim\left\|\left(\int_{0}^{1}\left(t^{-\alpha / 2}\left|W_{t}^{(1)} f(x)\right|\right)^{q} \frac{d t}{t}\right)^{1 / q}\right\|_{L^{p}\left(\mu_{\chi}\right)} \\
& \lesssim\|f\|_{F_{\alpha}^{p, q}}
\end{aligned}
$$

Next, using (33) and applying [6, Proposition 3.5] and then arguing as before, we estimate

$$
\begin{aligned}
\left\|\Sigma_{1}^{1 / q}\right\|_{L^{p}\left(\mu_{\chi}\right)} & \lesssim\left\|\left(\sum_{m=1}^{+\infty}\left(2^{m \alpha / 2} e^{-c 2^{-m} \Delta_{\chi}} g_{m}\right)^{q}\right)^{1 / q}\right\|_{L^{p}\left(\mu_{\chi}\right)} \\
& \lesssim\left\|\left(\sum_{m=1}^{+\infty}\left(2^{m \alpha / 2} g_{m}\right)^{q}\right)^{1 / q}\right\|_{L^{p}\left(\mu_{\chi}\right)} \\
& \lesssim\left\|\left(\sum_{m=1}^{+\infty} 2^{m \alpha q / 2-m(q-1)} \int_{2^{-m-1}}^{2^{-m}}\left|\frac{\partial}{\partial t} e^{-t \Delta_{\chi}} f\right|^{q} d t\right)^{1 / q}\right\|_{L^{p}\left(\mu_{\chi}\right)} \\
& \lesssim\|f\|_{F_{\alpha}^{p, q}} .
\end{aligned}
$$

This completes Step 2.

Step 3 We finish the proof by showing that

$$
\left\|\mathbb{S}_{\alpha}^{\text {loc, } q} e^{-\Delta_{\chi}} f\right\|_{L^{p}\left(\mu_{\chi}\right)} \lesssim\|f\|_{L^{p}\left(\mu_{\chi}\right)} .
$$

We first notice that for every $x \in G$ and $y \in B_{1}$,

$$
\begin{aligned}
\left|\mathrm{D}_{y}\left(e^{-\Delta_{\chi}} f\right)(x)\right| & \lesssim|y| \sup \left\{\left|X_{i} e^{-\Delta_{\chi}} f(w)\right|:\left|w^{-1} x\right| \leq|y|, i=1, \ldots, \ell\right\} \\
& \lesssim|y| \sup \left\{\left|X_{i} e^{-\Delta_{\chi}} f(w)\right|:\left|w^{-1} x\right| \leq 1, i=1, \ldots, \ell\right\} .
\end{aligned}
$$


By Lemma 1 there exists $t_{0}>0$ such that for every $w$ such that $\left|w^{-1} x\right| \leq 1$, and $i=1, \ldots, \ell$,

$$
\begin{aligned}
\left|X_{i} e^{-\Delta_{\chi}} f(w)\right| & =\left|f * X_{i} p_{1}^{\chi}(w)\right| \\
& \leq \int\left|f\left(w y^{-1}\right)\right|\left|X_{i} p_{1}^{\chi}(y)\right| d \rho(y) \\
& \lesssim \int\left|f\left(w y^{-1}\right)\right|\left(\delta \chi^{-1}\right)^{1 / 2}(y) e^{-c|y|^{2}} d \rho(y) \\
& \lesssim \int|f(z)|\left(\delta \chi^{-1}\right)^{1 / 2}\left(z^{-1} w\right) e^{-c\left|z^{-1} w\right|^{2}} d \lambda(z) \\
& \lesssim \int|f(z)| p_{t_{0}}^{\chi}\left(z^{-1} x\right) d \lambda(z) \\
& =e^{-t_{0} \Delta_{\chi}}|f|(x) .
\end{aligned}
$$

Therefore,

$$
\begin{aligned}
\left(\mathbb{S}_{\alpha}^{\operatorname{loc}, q}\left(e^{-\Delta_{\chi}} f(x)\right)\right)^{q} & =\int_{0}^{1}\left(\frac{1}{u^{\alpha} V(u)} \int_{|y|<u}\left|\mathrm{D}_{y}\left(e^{-\Delta_{\chi}} f\right)(x)\right| d \rho(y)\right)^{q} \frac{d u}{u} \\
& \lesssim \int_{0}^{1}\left(\frac{1}{u^{\alpha} V(u)} \int_{|y|<u} u e^{-t_{0} \Delta_{\chi}}|f|(x) d \rho(y)\right)^{q} \frac{d u}{u} \\
& \lesssim e^{-t_{0} \Delta_{\chi}}|f|(x)^{q}
\end{aligned}
$$

where we used the fact that $\alpha \in(0,1)$. Hence,

$$
\left\|\mathbb{S}_{\alpha}^{\mathrm{loc}, q} e^{-\Delta_{\chi}} f\right\|_{L^{p}\left(\mu_{\chi}\right)} \lesssim\left\|e^{-t_{0} \Delta_{\chi}}|f|\right\|_{L^{p}\left(\mu_{\chi}\right)} \lesssim\|f\|_{L^{p}\left(\mu_{\chi}\right)}
$$

which completes Step 3, and the proof of the theorem.

\subsection{Characterization of Besov Norm by Differences}

We now prove a characterization of the Besov norm in terms of the difference operator (16). Its proof is inspired by the one of [9, Theorem 1.16], in the case of a sub-Laplacian without drift on a unimodular group $G$ with respect to the Haar measure.

We set

$$
\mathbb{A}_{\alpha}^{p, q}(f)=\left(\int_{|y| \leq 1}\left(\frac{\left\|\mathrm{D}_{y} f\right\|_{L^{p}\left(\mu_{\chi}\right)}}{|y|^{\alpha}}\right)^{q} \frac{d \rho(y)}{V(|y|)}\right)^{1 / q},
$$


Theorem 9 Let $\alpha \in(0,1)$ and $p, q \in[1,+\infty]$. Then

$$
\|f\|_{B_{\alpha}^{p, q}} \approx \mathbb{A}_{\alpha}^{p, q}(f)+\|f\|_{L^{p}\left(\mu_{\chi}\right)}
$$

for any $f \in B_{\alpha}^{p, q}\left(\mu_{\chi}\right)$.

Proof We separate the proof in three steps. The first step deals with some simple integral estimates relying on the classical Schur's test, while the second and third steps contain the inequalities $\lesssim$ and $\gtrsim$, respectively, in the statement.

Step 1 Let $a \in \mathbb{R}, s \geq 0, c>0$ and define the integral kernel $K:(0,1) \times G \rightarrow$ $[0,+\infty)$ by

$$
K(t, y)=\chi^{a}(y)\left(\frac{|y|^{2}}{t}\right)^{s} \frac{V(|y|)}{V(\sqrt{t})} e^{-c|y|^{2} / t},
$$

and the corresponding integral operator

$$
T_{K} g(t)=\int_{G} K(t, y) g(y) \frac{d \rho(y)}{V(|y|)} .
$$

Then, we show that for all $q \in[1,+\infty)$

$$
T_{K}: L^{q}(G, d \rho / V(|\cdot|)) \rightarrow L^{q}((0,1), d t / t)
$$

is bounded.

To this end, it suffices to apply Schur's test, see e.g. [11, Theorem 6.18], after showing that

$$
\text { (a) } \int_{G} K(t, y) \frac{d \rho(y)}{V(|y|)} \lesssim 1, \quad \text { (b) } \int_{0}^{1} K(t, y) \frac{d t}{t} \lesssim 1 .
$$

Observe that

$$
\int_{G} K(t, y) \frac{d \rho(y)}{V(|y|)}=\int_{|y|^{2} \leq t} K(t, y) \frac{d \rho(y)}{V(|y|)}+\int_{|y|^{2}>t} K(t, y) \frac{d \rho(y)}{V(|y|)}=: I+I I .
$$

It is easy to check that $I \lesssim 1$. Moreover, since by (2) for every $j \geq 0$ and $t \in(0,1)$

$$
\frac{V\left(2^{j+1} \sqrt{t}\right)}{V(\sqrt{t})} \lesssim 2^{d(j+1)} e^{D 2^{j+1}}
$$

we have

$$
I I \lesssim \sum_{j=0}^{\infty} \int_{2^{j} \sqrt{t} \leq|y|<2^{j+1} \sqrt{t}} \frac{1}{V(\sqrt{t})} 2^{2 j s} e^{a c_{X} 2^{j+1} \sqrt{t}} e^{-c 2^{2 j}} d \rho(y) \lesssim 1
$$


Thus, condition (a) in (37) is satisfied. In order to prove (b), we separate two cases. If $|y| \geq 1$, then

$$
\int_{0}^{1} K(t, y) \frac{d t}{t} \lesssim e^{a c_{X}|y|-\frac{c}{2}|y|^{2}} V(|y|) \int_{0}^{1} \frac{1}{V(\sqrt{t})} e^{-\frac{c}{4}|y|^{2} / t} \frac{d t}{t} \lesssim 1
$$

while, if $|y| \leq 1$, (hence $\chi(y) \lesssim 1$ )

$$
\int_{0}^{1} K(t, y) \frac{d t}{t} \lesssim \int_{0}^{1}\left(\frac{|y|^{2}}{t}\right)^{s+d / 2} e^{-c|y|^{2} / t} \frac{d t}{t}=\int_{|y|^{2}}^{\infty} u^{s+d / 2} e^{-c u} \frac{d u}{u} \lesssim 1,
$$

which proves (37). This completes Step 1.

Step 2 We show that, for $p, q \in[1,+\infty]$ and $\alpha>0$,

$$
\mathbb{B}_{\alpha}^{p, q}(f) \lesssim \mathbb{A}_{\alpha}^{p, q}(f)+\|f\|_{L^{p}\left(\mu_{\chi}\right)}
$$

We claim that there exists $c>0$ such that, for all $f \in L^{p}\left(\mu_{\chi}\right)$,

$$
\mathbb{B}_{\alpha}^{p, q}(f) \lesssim\left(\int_{G}\left(\frac{\left\|\mathrm{D}_{y} f\right\|_{L^{p}\left(\mu_{\chi}\right)} e^{-c|y|^{2}}}{|y|^{\alpha}}\right)^{q} \frac{d \rho(y)}{V(|y|)}\right)^{1 / q}
$$

Assuming the claim, we prove the estimate (38). By the claim, it suffices to prove that

$$
\left(\int_{G}\left(\frac{\left\|\mathrm{D}_{y} f\right\|_{L^{p}\left(\mu_{\chi}\right)} e^{-c|y|^{2}}}{|y|^{\alpha}}\right)^{q} \frac{d \rho(y)}{V(|y|)}\right)^{1 / q} \lesssim \mathbb{A}_{\alpha}^{p, q}(f)+\|f\|_{L^{p}\left(\mu_{\chi}\right)} .
$$

We split the integral on $G$ as $\{|y|<1\} \cup\{|y| \geq 1\}$. On the one hand, it is easy to see that

$$
\left(\int_{|y| \leq 1}\left(\frac{\left\|\mathrm{D}_{y} f\right\|_{L^{p}\left(\mu_{\chi}\right)} e^{-c|y|^{2}}}{|y|^{\alpha}}\right)^{q} \frac{d \rho(y)}{V(|y|)}\right)^{1 / q} \leq \mathbb{A}_{\alpha}^{p, q}(f)
$$

while since $\left\|\mathrm{D}_{y} f\right\|_{L^{p}\left(\mu_{\chi}\right)} \leq\left(1+\chi^{1 / p}(y)\right)\|f\|_{L^{p}\left(\mu_{\chi}\right)}$,

$$
\begin{aligned}
& \left(\int_{|y| \geq 1}\left(\frac{\left\|\mathrm{D}_{y} f\right\|_{L^{p}\left(\mu_{\chi}\right)} e^{-c|y|^{2}}}{|y|^{\alpha}}\right)^{q} \frac{d \rho(y)}{V(|y|)}\right)^{1 / q} \\
& \lesssim\|f\|_{L^{p}\left(\mu_{\chi}\right)}\left(\int_{|y| \geq 1}\left(1+e^{c_{X}|y|}\right) e^{-c q|y|^{2}} d \rho(y)\right)^{1 / q} \\
& \lesssim\|f\|_{L^{p}\left(\mu_{\chi}\right)}
\end{aligned}
$$

since the volume of balls grows at most exponentially (see (2)). 
It remains to prove the claim (39). Since

$$
\int_{G} \partial_{t} p_{t}^{\chi}(y) d \rho(y)=\partial_{t} \int_{G} p_{t}^{\chi}(y) d \rho(y)=0,
$$

we have

$$
\begin{aligned}
\frac{\partial}{\partial t} e^{-t \Delta_{\chi}} f(x) & =\int_{G} f\left(x y^{-1}\right) \frac{\partial p_{t}^{\chi}}{\partial t}(y) d \rho(y) \\
& =\int_{G} \frac{\partial p_{t}^{\chi}}{\partial t}(y) \mathrm{D}_{y} f(x) d \rho(y)
\end{aligned}
$$

Thus,

$$
\left\|\frac{\partial}{\partial t} e^{-t \Delta_{\chi}} f\right\|_{L^{p}\left(\mu_{\chi}\right)} \leq \int_{G}\left|\frac{\partial p_{t}^{\chi}}{\partial t}(y)\right|\left\|\mathrm{D}_{y} f\right\|_{L^{p}\left(\mu_{\chi}\right)} d \rho(y) .
$$

By Lemma 1 (v), (40) and [6, Lemma 3.3]

$$
\begin{aligned}
& \mathbb{B}_{\alpha}^{p, q}(f)^{q} \\
& \approx \int_{0}^{1}\left(t^{1-\alpha / 2}\left\|\frac{\partial}{\partial t} e^{-t \Delta_{\chi}} f\right\|_{L^{p}\left(\mu_{\chi}\right)}\right)^{q} \frac{d t}{t} \\
& \lesssim \int_{0}^{1}\left(t^{1-\alpha / 2} \int_{G}\left(\delta \chi^{-1}\right)^{1 / 2}(y) t^{-(d+2) / 2} e^{-b|y|^{2} / t}\left\|\mathrm{D}_{y} f\right\|_{L^{p}\left(\mu_{x}\right)} d \rho(y)\right)^{q} \frac{d t}{t} \\
& \lesssim \int_{0}^{1}\left(t^{-\alpha / 2} \int_{G}\left(\delta \chi^{-1}\right)^{1 / 2}(y) t^{-d / 2} e^{-b^{\prime}|y|^{2} / t}\left\|\mathrm{D}_{y} f\right\|_{L^{p}\left(\mu_{x}\right)} e^{-b^{\prime}|y|^{2}} d \rho(y)\right)^{q} \frac{d t}{t} \\
& =\int_{0}^{1}\left(\int_{G} K(t, y) g(y) \frac{d \rho(y)}{V(|y|)}\right)^{q} \frac{d t}{t}
\end{aligned}
$$

with

$$
\begin{gathered}
b^{\prime}=\frac{b}{2}, \quad g(y)=\frac{\left\|\mathrm{D}_{y} f\right\|_{L^{p}\left(\mu_{\chi}\right)}}{|y|^{\alpha}} e^{-b^{\prime}|y|^{2}}, \\
K(t, y)=\frac{V(|y|)}{t^{d / 2}}\left(\frac{|y|^{2}}{t}\right)^{\alpha / 2}\left(\delta \chi^{-1}\right)^{1 / 2}(y) e^{-b^{\prime}|y|^{2} / t} .
\end{gathered}
$$

By Step 1 we obtain

$$
\mathbb{B}_{\alpha}^{p, q}(f)^{q} \lesssim \int_{G}|g(y)|^{q} \frac{d \rho(y)}{V(|y|)}=\int_{G}\left(\frac{\left\|\mathrm{D}_{y} f\right\|_{L^{p}\left(\mu_{\chi}\right)} e^{-b^{\prime}|y|^{2}}}{|y|^{\alpha}}\right)^{q} \frac{d \rho(y)}{V(|y|)} .
$$

The claim is proved, and Step 2 is complete. 
Step 3 We prove that, for $p, q \in[1,+\infty]$ and $\alpha \in(0,1)$,

$$
\mathbb{A}_{\alpha}^{p, q}(f) \lesssim \mathbb{B}_{\alpha}^{p, q}(f)+\|f\|_{L^{p}\left(\mu_{\chi}\right)}
$$

We write again $f$ as $f=\left(f-e^{-\Delta_{\chi}} f\right)+e^{-\Delta_{\chi}} f$ and decompose $f-e^{-\Delta_{\chi}} f=$ $\sum_{m=1}^{\infty} f_{m}$ as in (25). Then, using also (30), we have

$$
\begin{aligned}
f_{m} & =\int_{2^{-m}}^{2^{-m+1}} \frac{\partial}{\partial t} e^{-t \Delta_{\chi}} f d t=\int_{2^{-m}}^{2^{-m+1}} \Delta_{\chi} e^{-t \Delta_{\chi}} f d t=2 \int_{2^{-m-1}}^{2^{-m}} \Delta_{\chi} e^{-2 t \Delta_{\chi}} f d t \\
& =2 e^{-2^{-m-1} \Delta_{\chi}} \int_{2^{-m-1}}^{2^{-m}} e^{-\left(t-2^{-m-1}\right) \Delta_{\chi}} \Delta_{\chi} e^{-t \Delta_{\chi}} f d t=: 2 e^{-2^{-m-1} \Delta_{\chi}} h_{m} .
\end{aligned}
$$

We set

$$
\sigma_{m}=\int_{2^{-m-1}}^{2^{-m}}\left\|\frac{\partial}{\partial t} e^{-t \Delta_{\chi}} f\right\|_{L^{p}\left(\mu_{\chi}\right)} d t
$$

and observe that

$$
\left\|f_{m}\right\|_{L^{p}\left(\mu_{\chi}\right)} \lesssim \sigma_{m+1}
$$

Hence,

$$
\left\|\mathrm{D}_{y} f_{m}\right\|_{L^{p}\left(\mu_{\chi}\right)} \lesssim\left(1+\chi^{1 / p}(y)\right)\left\|f_{m}\right\|_{L^{p}\left(\mu_{\chi}\right)} \lesssim \sigma_{m+1}
$$

By $[6$, Lemma 3.3] it follows that for $i=1, \ldots, \ell$,

$$
\begin{aligned}
\left\|X_{i} f_{m}\right\|_{L^{p}\left(\mu_{\chi}\right)} & \lesssim 2^{m / 2}\left\|h_{m}\right\|_{L^{p}\left(\mu_{\chi}\right)} \\
& \lesssim 2^{m / 2} \int_{2^{-m-1}}^{2^{-m}}\left\|e^{-\left(t-2^{-m-1}\right) \Delta_{\chi}} \frac{\partial}{\partial t} e^{-t \Delta_{\chi}} f\right\|_{L^{p}\left(\mu_{\chi}\right)} d t \lesssim 2^{m / 2} \sigma_{m} .
\end{aligned}
$$

Therefore,

$$
\left\|\mathrm{D}_{y} f_{m}\right\|_{L^{p}\left(\mu_{\chi}\right)} \lesssim|y| \sum_{i=1}^{\ell}\left\|X_{i} f_{m}\right\|_{L^{p}\left(\mu_{\chi}\right)} \lesssim|y| 2^{m / 2} \sigma_{m}
$$

Since $\sigma_{m} \leq 2 \sigma_{m+1},(43),(41)$ and (42) imply

$$
\left\|\mathrm{D}_{y} f_{m}\right\|_{L^{p}\left(\mu_{\chi}\right)} \lesssim \begin{cases}|y| 2^{m / 2} \sigma_{m} & \text { if }|y|^{2}<2^{-m} \\ \sigma_{m+1} & \text { if }|y|^{2} \geq 2^{-m}\end{cases}
$$


Therefore, by Lemma 4.3 (i) in [6], we have

$$
\begin{aligned}
\left(\mathbb{A}_{\alpha}^{p, q}\left(f-e^{-\Delta_{\chi}} f\right)\right)^{q} & \lesssim \sum_{j=1}^{\infty} \int_{2^{-j} \leq|y|^{2}<2^{-j+1}} 2^{j q \alpha / 2}\left(\sum_{m=1}^{\infty}\left\|\mathrm{D}_{y} f_{m}\right\|_{L^{p}\left(\mu_{\chi}\right)}\right)^{q} \frac{d \rho(y)}{V(|y|)} \\
& \lesssim \sum_{j=1}^{\infty} 2^{j q \alpha / 2}\left(\sum_{m=1}^{j} 2^{(m-j) / 2} \sigma_{m}+\sum_{m=j+1}^{+\infty} \sigma_{m+1}\right)^{q} \\
& \lesssim \sum_{j=1}^{\infty} 2^{j q(\alpha-1) / 2}\left(\sum_{m=1}^{\infty} 2^{\min \{j, m\} / 2}\left(\sigma_{m+1}+\sigma_{m}\right)\right)^{q} \\
& \lesssim \sum_{m=1}^{\infty}\left(2^{m \alpha / 2}\left(\sigma_{m+1}+\sigma_{m}\right)\right)^{q} \\
& \lesssim \sum_{m=0}^{\infty}\left(2^{m \alpha / 2} \int_{2^{-m-1}}^{2^{-m}}\left\|\frac{\partial}{\partial t} e^{-t \Delta_{\chi}} f\right\|_{L^{p}\left(\mu_{\chi}\right)} d t\right)^{q} \\
& \lesssim \sum_{m=0}^{\infty} 2^{m q \alpha / 2} 2^{-m(q-1)} \int_{2^{-m-1}}^{2^{-m}}\left\|\frac{\partial}{\partial t} e^{-t \Delta_{\chi}} f\right\|_{L^{p}\left(\mu_{\chi}\right)}^{q} d t \\
& \lesssim \sum_{m=0}^{\infty} \int_{2^{-m-1}}^{2^{-m}}\left(t^{1-\alpha / 2}\left\|\frac{\partial}{\partial t} e^{-t \Delta_{\chi}} f\right\|_{L^{p}\left(\mu_{\chi}\right)}\right)^{q} \frac{d t}{t} \\
& =\left(\mathbb{B}_{\alpha}^{p, q}(f)\right)^{q} .
\end{aligned}
$$

Therefore, by (9) we have

$$
\mathbb{A}_{\alpha}^{p, q}\left(f-e^{-\Delta_{\chi}} f\right) \lesssim \mathbb{B}_{\alpha}^{p, q}(f)
$$

It remains to estimate $\mathbb{A}_{\alpha}^{p, q}\left(e^{-\Delta_{\chi}} f\right)$. As in (43), one can see that

$$
\left\|\mathrm{D}_{y} e^{-\Delta_{\chi}} f\right\|_{L^{p}\left(\mu_{\chi}\right)} \lesssim|y|\|f\|_{L^{p}\left(\mu_{\chi}\right)}
$$

so that, using the decomposition of the integral as sum of integrals over annuli,

$$
\mathbb{A}_{\alpha}^{p, q}\left(e^{-\Delta_{\chi}} f\right) \leq\|f\|_{L^{p}\left(\mu_{\chi}\right)}\left(\int_{|y| \leq 1}|y|^{q(1-\alpha)} \frac{d \rho(y)}{V(|y|)}\right)^{1 / q} \lesssim\|f\|_{L^{p}\left(\mu_{\chi}\right)}
$$

The proof of Step 3 is complete. This concludes the proof of Theorem 9. 


\section{A Density Result}

The main goal of this section is to show that the smooth functions with compact support are dense in the Triebel-Lizorkin and Besov spaces on $G$. This is the analogue of the classical density result in the Euclidean setting [53]; we refer the reader to $[22,24,52]$ for its counterpart in other settings.

To prove our density results we shall use the following version of Young's inequality which follows from [4, Proposition 12 of Chapter VIII, §4, No. 5].

Lemma 10 If $\eta$ has support in $B_{1}$, then

$$
\|\eta * f\|_{L^{p}\left(\mu_{\chi}\right)} \lesssim\|\eta\|_{L^{1}\left(\mu_{\chi}\right)}\|f\|_{L^{p}\left(\mu_{\chi}\right)}
$$

for any $f \in L^{p}\left(\mu_{\chi}\right)$.

We now prove the density result.

Theorem 11 Let $\alpha>0, p, q \in(1, \infty)$ and let $X_{\alpha}^{p, q}$ denote either $F_{\alpha}^{p, q}\left(\mu_{\chi}\right)$ or $B_{\alpha}^{p, q}\left(\mu_{\chi}\right)$. Then, $C_{c}^{\infty}(G)$ is dense in $X_{\alpha}^{p, q}$.

Proof We begin by observing that Lemma 3 implies that $\mathcal{S}$, hence $C_{c}^{\infty}$, is contained in $X_{\alpha}^{p, q}$. Indeed, if $m, n \in \mathbb{N}$ with $m>[\alpha / 2]$ and $n$ to be chosen, for $\varphi \in \mathcal{S}$, using (7) we have

$$
\left|W_{t}^{(m)} \varphi(x)\right| \leq t^{m} e^{-n|x|} \sum_{|J| \leq 2 m} \mathcal{N}_{J, n}(\varphi)
$$

Then, in order to estimate the norms in (10) and (11), it suffices to chose $n$ large enough so that $\int_{G} e^{-p n|x|} d \mu_{\chi}(x)$ is finite.

Step 1 We first prove that we can approximate functions in $F_{\alpha}^{p, q}$ with functions having compact support when $\alpha \in(0,1)$.

Let $\eta \in C_{c}^{\infty}\left(B_{1}\right), \eta \geq 0, \int_{G} \eta d \rho=1$. Given any $R>2$, define $\eta_{R}=\mathbf{1}_{B_{R}} * \eta$. Then, $\eta_{R} \in C_{c}^{\infty}\left(B_{R+1}\right)$ and $\eta_{R}(x)=1$ on $B_{R-1}$. We observe that $\left\|X_{J} \eta_{R}\right\|_{\infty} \lesssim 1$, for $|J| \leq n$. Indeed, the definition of the convolution implies that for any $J \in J^{k}$, with $k \leq n$, we have that for all $x \in G$

$$
\left|X_{J} \eta_{R}(x)\right| \leq \int_{G} \mathbf{1}_{B_{R}}\left(y^{-1}\right)\left|X_{J} \eta(y x)\right| d \rho(y) \leq\left\|X_{J} \eta\right\|_{L^{1}(\rho)} \lesssim 1 .
$$

Hence

$$
\left\|X_{J} \eta_{R}\right\|_{\infty} \lesssim 1
$$

Then, let $f \in F_{\alpha}^{p, q}$ be given. We shall estimate the norm $\left\|f-f \eta_{R}\right\|_{F_{\alpha}^{p, q}}$ using the $\mathbb{S}_{\alpha}^{\text {loc, } q}$-functional. Since $\alpha \in(0,1), f \in L^{p}\left(\mu_{\chi}\right)$ and $\left\|f-f \eta_{R}\right\|_{L^{p}\left(\mu_{\chi}\right)} \rightarrow 0$ as 
$R \rightarrow+\infty$. Next, we show that also

$$
\left\|\mathbb{S}_{\alpha}^{\operatorname{loc}, q}\left(f-f \eta_{R}\right)\right\|_{L^{p}\left(\mu_{\chi}\right)} \rightarrow 0
$$

as $R \rightarrow+\infty$. Set $\zeta_{R}=1-\eta_{R}$ and observe that $\zeta_{R}$ vanishes identically on $B_{R-1}$. Then, we have

$$
\begin{aligned}
& {\left[\mathbb{S}_{\alpha}^{\operatorname{loc}, q}\left(f-f \eta_{R}\right)(x)\right]^{q}} \\
& =\int_{0}^{1}\left[\frac{1}{u^{\alpha} V(u)} \int_{|y| \leq u}\left|\zeta_{R}\left(x y^{-1}\right) f\left(x y^{-1}\right)-\zeta_{R}(x) f(x)\right| d \rho(y)\right]^{q} \frac{d u}{u} \\
& \lesssim \int_{0}^{1}\left[\frac{1}{u^{\alpha} V(u)} \int_{|y| \leq u} \zeta_{R}\left(x y^{-1}\right)\left|f\left(x y^{-1}\right)-f(x)\right| d \rho(y)\right]^{q} \frac{d u}{u} \\
& \quad+|f(x)|^{q} \int_{0}^{1}\left[\frac{1}{u^{\alpha} V(u)} \int_{|y| \leq u}\left|\zeta_{R}\left(x y^{-1}\right)-\zeta_{R}(x)\right| d \rho(y)\right]^{q} \frac{d u}{u} \\
& \lesssim \int_{0}^{1}\left[\frac{1}{u^{\alpha} V(u)} \int_{|y| \leq u} \zeta_{R}\left(x y^{-1}\right)\left|f\left(x y^{-1}\right)-f(x)\right| d \rho(y)\right]^{q} \frac{d u}{u} \\
& \quad+|f(x)|^{q} \int_{0}^{1}\left[\frac{1}{u^{\alpha} V(u)} \int_{|y| \leq u}|y| \sup _{z \in B(x, 1)} \sum_{j=1}^{\ell}\left|X_{j} \zeta_{R}(z)\right| d \rho(y)\right]^{q} \frac{d u}{u} \\
& \left.\left.\lesssim \mathbf{1}_{\{|x| \geq R-2\}(x)\left(\left[\mathbb{S}_{\alpha} \operatorname{loc}, q\right.\right.} f(x)\right]^{q}+|f(x)|^{q}\right),
\end{aligned}
$$

since $\zeta_{R}(z)=0$ if $|z| \leq R-1$, and $\left\|\zeta_{R}\right\|_{\infty}=1$. Therefore,

$$
\begin{aligned}
\| \mathbb{S}_{\alpha}^{\mathrm{loc}, q}(f- & \left.f \eta_{R}\right) \|_{L^{p}\left(\mu_{\chi}\right)}^{p} \\
& \lesssim \int_{\{|x| \geq R-2\}}\left[\mathbb{S}_{\alpha}^{\operatorname{loc}, q} f(x)\right]^{p} d \mu_{\chi}(x)+\int_{\{|x| \geq R-2\}}|f(x)|^{p} d \mu_{\chi}(x) .
\end{aligned}
$$

This proves (46) and therefore we can approximate any element of $F_{\alpha}^{p, q}$ with elements with compact support.

Step 2 Using the characterization of the norm in $B_{\alpha}^{p, q}$ by finite differences for $\alpha \in(0,1)$, Theorem 9 , we prove that we can approximate functions in $B_{\alpha}^{p, q}$ with functions with compact support.

Let $f \in B_{\alpha}^{p, q}$ be given and let $\eta_{R}$ and $\zeta_{R}$ be as in Step 1. Then, for $y \in B_{1}$ and $x \in G$ we write

$$
\begin{aligned}
\mathrm{D}_{y}\left(f \zeta_{R}\right)(x) & =\left(f \zeta_{R}\right)\left(x y^{-1}\right)-\left(f \zeta_{R}\right)(x) \\
& =\zeta_{R}\left(x y^{-1}\right)\left[f\left(x y^{-1}\right)-f(x)\right]+f(x)\left[\zeta_{R}\left(x y^{-1}\right)-\zeta_{R}(x)\right] \\
& =: F_{R}(x, y)+G_{R}(x, y) .
\end{aligned}
$$


We observe that

$$
\begin{aligned}
& \left(\int_{|y| \leq 1}\left(\frac{\left\|G_{R}(\cdot, y)\right\|_{L^{p}\left(\mu_{\chi}\right)}}{|y|^{\alpha}}\right)^{q} \frac{d \rho(y)}{V(|y|)}\right)^{1 / q} \\
& \lesssim\left(\int_{|y| \leq 1}|y|^{(1-\alpha) q} \frac{d \rho(y)}{V(|y|)}\right)^{1 / q}\left\|\mathbf{1}_{\{|x| \geq R-2\}} f\right\|_{L^{p}\left(\mu_{\chi}\right)} \\
& \lesssim\left\|\mathbf{1}_{\{|x| \geq R-2\}} f\right\|_{L^{p}\left(\mu_{\chi}\right)},
\end{aligned}
$$

that tends to 0 as $R \rightarrow+\infty$. Next, we observe that

$$
\left|F_{R}(x, y)\right| \leq \mathbf{1}_{\{|x| \geq R-2\}}\left|\mathrm{D}_{y} f(x)\right| \leq\left|\mathrm{D}_{y} f(x)\right|,
$$

so that

- $\left\|F_{R}(\cdot, y)\right\|_{L^{p}\left(\mu_{\chi}\right)} \leq\left(\int_{\{|x| \geq R-2\}}\left|\mathrm{D}_{y} f(x)\right|^{p} d \mu_{\chi}(x)\right)^{1 / p} \rightarrow 0$, as $R \rightarrow+\infty$;

- $\left\|F_{R}(\cdot, y)\right\|_{L^{p}\left(\mu_{\chi}\right)} \leq\left\|\mathrm{D}_{y} f\right\|_{L^{p}\left(\mu_{\chi}\right)}$, which is independent of $R$.

Lebesgue's theorem now gives that

$$
\left(\int_{|y| \leq 1}\left(\frac{\left\|F_{R}(\cdot, y)\right\|_{L^{p}\left(\mu_{\chi}\right)}}{|y|^{\alpha}}\right)^{q} \frac{d \rho(y)}{V(|y|)}\right)^{1 / q} \rightarrow 0
$$

as $R \rightarrow+\infty$. Hence, recalling (35), we have

$$
\begin{aligned}
\mathbb{A}_{\alpha}^{p, q}\left(f-f \eta_{R}\right) \leq\left(\int_{|y| \leq 1}\right. & \left.\left(\frac{\left\|F_{R}(\cdot, y)\right\|_{L^{p}\left(\mu_{\chi}\right)}}{|y|^{\alpha}}\right)^{q} \frac{d \rho(y)}{V(|y|)}\right)^{1 / q} \\
& +\left(\int_{|y| \leq 1}\left(\frac{\left\|G_{R}(\cdot, y)\right\|_{L^{p}\left(\mu_{\chi}\right)}}{|y|^{\alpha}}\right)^{q} \frac{d \rho(y)}{V(|y|)}\right)^{1 / q} \rightarrow 0,
\end{aligned}
$$

as $R \rightarrow+\infty$. This completes Step 2 .

Step 3 We select a smooth approximation of the identity. Precisely, for $0<\kappa \leq$ $1 / 2$, select $\psi_{\kappa} \in C_{c}^{\infty}\left(B_{\kappa}\right), \psi_{\kappa} \geq 0$, and $\left\|\psi_{\kappa}\right\|_{L^{1}(\lambda)}=1$. Moreover, we require that $\psi_{\kappa} \lesssim V(2 \kappa)^{-1}$ (where the constant does not depend on $\left.\kappa\right){ }^{2}$ We then have

$$
\left|\psi_{\kappa} * f(x)\right| \lesssim M f(x)
$$

\footnotetext{
${ }^{2}$ This can achieved as follows. Let $\tilde{\psi} \in C_{c}^{\infty}, 0 \leq \tilde{\psi} \leq 1$, supp $\tilde{\psi} \subseteq B_{\kappa / 2}, \int_{G} \tilde{\psi} d \rho=1$, and define $\psi_{\kappa}=C_{\kappa} V(2 \kappa)^{-1} \mathbf{1}_{B_{\kappa / 4}} * \tilde{\psi}$. By requiring that $\left\|\psi_{\kappa}\right\|_{L^{1}(\lambda)}=1$, we obtain that $C_{\kappa} \approx 1$.
} 
where $M$ is the local maximal function, defined in (28). Indeed, we estimate

$$
\left|\psi_{\kappa} * f(x)\right|=\left|\int_{G} \psi_{\kappa}\left(x y^{-1}\right) f(y) d \rho(y)\right| \lesssim \frac{1}{V(2 \kappa)} \int_{B(x, \kappa)}|f(y)| d \rho \lesssim M f(x),
$$

as claimed.

By [4, Proposition 20 of Chapter VIII, §4, No. 7] for any $g \in L^{p}\left(\mu_{\chi}\right), \| \psi_{\kappa} * g-$ $g \|_{L^{p}\left(\mu_{\chi}\right)}$ tends to 0 , as $\kappa \rightarrow 0$. Next, notice that, by left invariance, $W_{t}^{(m)}\left(\psi_{\kappa} * f\right)=$ $\psi_{\kappa} * W_{t}^{(m)} f$. Moreover, if $f \in \mathcal{S}^{\prime}$ has compact support, then $\psi_{\kappa} * f \in C_{c}^{\infty}(G)$.

Step 4 We complete the proof that $C_{c}^{\infty}$ is dense in the Triebel-Lizorkin spaces, $F_{\alpha}^{p, q}$ in the case $\alpha \in(0,1)$. To this end, let $f \in F_{\alpha}^{p, q}$ have compact support so that $\psi_{\kappa} * f \in C_{c}^{\infty}$.

Given an integer $m>\alpha / 2$, by Theorem 7 we have

$$
\begin{aligned}
& \left\|\psi_{\kappa} * f-f\right\|_{F_{\alpha}^{p, q}} \\
\lesssim & \left\|\left(\sum_{j=0}^{+\infty}\left(2^{j \alpha / 2}\left|\psi_{\kappa} * W_{2^{-j}}^{(m)} f-W_{2^{-j}}^{(m)} f\right|\right)^{q}\right)^{1 / q}\right\|_{L^{p}\left(\mu_{\chi}\right)}+\left\|\psi_{\kappa} * f-f\right\|_{L^{p}\left(\mu_{\chi}\right)} .
\end{aligned}
$$

We only need to estimate the first term in the right hand side above. Observe that, since $f$ has compact support, $W_{t}^{(m)} f \in \mathcal{S}$, for all $t \in(0,1)$. Hence, [12, Proposition 2.44] gives that, for each $j$ fixed

$$
\left\|\psi_{\kappa} * W_{2^{-j}}^{(m)} f-W_{2^{-j}}^{(m)} f\right\|_{\infty} \rightarrow 0 \quad \text { as } \quad \kappa \rightarrow 0
$$

We wish to apply Lebesgue's theorem to the inner sum. We observe that by (47) we have that

$$
\left(2^{j \alpha / 2}\left|\psi_{\kappa} * W_{2^{-j}}^{(m)} f-W_{2^{-j}}^{(m)} f\right|\right)^{q} \lesssim\left(2^{j \alpha / 2}\left[M\left(W_{2^{-j}}^{(m)} f\right)+\left|W_{2^{-j}}^{(m)} f\right|\right]\right)^{q}
$$

which is (independent of $\kappa$ and) summable by [17, p. 481]. Thus, the inner sum tends to 0 , as $\kappa \rightarrow 0$, for every $x \in G$, that is, the family of vector-valued functions $S_{\kappa}$,

$$
S_{\kappa}:=\left(2^{j \alpha / 2} \psi_{\kappa} * W_{2^{-j}}^{(m)} f\right)_{j}: G \rightarrow \ell^{q}
$$

as $\kappa \rightarrow 0$ converges pointwise to the vector-valued function $S: G \rightarrow \ell^{q}$, where $S:=\left(2^{j \alpha / 2} W_{2^{-j}}^{(m)} f\right)_{j}$. Since, as before, for $0<\kappa \leq 1 / 2$,

$$
\left\|S_{\kappa}(x)\right\|_{\ell q} \lesssim\left(\sum_{j=0}^{+\infty}\left(2^{j \alpha / 2} M\left(W_{2^{-j}}^{(m)} f(x)\right)^{q}\right)^{1 / q} \in L^{p}\left(\left(G, \ell^{q}\right), \mu_{\chi}\right) .\right.
$$


We can apply Lebesgue's theorem to obtain that $S_{\kappa} \rightarrow S$ in $L^{p}\left(\left(G, \ell^{q}\right), \mu_{\chi}\right)$, that is,

$$
\left\|\left(\sum_{j=0}^{+\infty}\left(2^{j \alpha / 2}\left|\psi_{\kappa} * W_{2^{-j}}^{(m)} f-W_{2^{-j}}^{(m)} f\right|\right)^{q}\right)^{1 / q}\right\|_{L^{p}\left(\mu_{\chi}\right)} \rightarrow 0 \quad \text { as } \quad \kappa \rightarrow 0,
$$

as we wished to show. Hence, $C_{c}^{\infty}$ is dense in $F_{\alpha}^{p, q}$, for $p, q \in(1, \infty), \alpha \in(0,1)$.

Step 5 We complete the proof that $C_{c}^{\infty}$ is dense in $B_{\alpha}^{p, q}$ in the case $\alpha \in(0,1)$. Let $f \in B_{\alpha}^{p, q}$ have compact support so that $\psi_{\kappa} * f \in C_{c}^{\infty}$. We then have

$$
\begin{aligned}
& \left\|\psi_{\kappa} * f-f\right\|_{B_{\alpha}^{p, q}} \\
\lesssim & \left(\int_{0}^{1}\left(t^{-\alpha / 2}\left\|\psi_{\kappa} * W_{t}^{(m)} f-W_{t}^{(m)} f\right\|_{L^{p}\left(\mu_{\chi}\right)}\right)^{q} \frac{d t}{t}\right)^{1 / q}+\left\|\psi_{\kappa} * f-f\right\|_{L^{p}\left(\mu_{\chi}\right)} .
\end{aligned}
$$

Now, $\left\|\psi_{\kappa} * f-f\right\|_{L^{p}\left(\mu_{\chi}\right)}$ and $\left\|\psi_{\kappa} * W_{t}^{(m)} f-W_{t}^{(m)} f\right\|_{L^{p}\left(\mu_{\chi}\right)} \rightarrow 0$, as $\kappa \rightarrow 0$, the latter term for each $t \in(0,1)$ fixed. Using Young's inequality (44) we see that

$$
\left\|\psi_{\kappa} * W_{t}^{(m)} f-W_{t}^{(m)} f\right\|_{L^{p}\left(\mu_{\chi}\right)} \lesssim\left\|W_{t}^{(m)} f\right\|_{L^{p}\left(\mu_{\chi}\right)},
$$

so that we may use Lebesgue's theorem to obtain that

$$
\left(\int_{0}^{1}\left(t^{-\alpha / 2}\left\|\psi_{\kappa} * W_{t}^{(m)} f-W_{t}^{(m)} f\right\|_{L^{p}\left(\mu_{x}\right)}\right)^{q} \frac{d t}{t}\right)^{1 / q} \rightarrow 0 \quad \text { as } \quad \kappa \rightarrow 0 .
$$

This gives that $\left\|\psi_{\kappa} * f-f\right\|_{B_{\alpha}^{p, q}} \rightarrow 0$, as $\kappa \rightarrow 0$, and completes the proof that $C_{c}^{\infty}$ is dense in $B_{\alpha}^{p, q}$, for $p, q \in(1, \infty), \alpha \in(0,1)$.

Step 6 We now prove that $C_{c}^{\infty}$ is dense in $X_{\alpha+1}^{p, q}$, for $p, q \in(1, \infty), \alpha \in(0,1)$. By [6, Theorem 4.5], $\|f\|_{X_{\alpha+1}^{p, q}} \approx\|f\|_{X_{\alpha}^{p, q}}+\sum_{j=1}^{\ell}\left\|X_{j} f\right\|_{X_{\alpha}^{p, q}}$ for every $f \in X_{\alpha+1}^{p, q}$. Let $\varepsilon>0$. By the arguments in Steps 1 and 2, using the same notation, there exists $R>0$ sufficiently large such that

$$
\left\|f-f \eta_{R}\right\|_{X_{\alpha}^{p, q}}<\varepsilon, \quad \text { and } \quad\left\|X_{j} f-\left(X_{j} f\right) \eta_{R}\right\|_{X_{\alpha}^{p, q}}<\varepsilon / 2 \text {, }
$$

for $j=1, \ldots, \ell$. Since $X_{j} \eta_{R}$ is a $C_{c}^{\infty}$ function vanishing in the ball $B_{R-1}$ and $X_{j} \eta_{R}$ are uniformly bounded by (45), by the arguments involving $\zeta_{R}$ in Steps 1 and 2 we can also assume that $\left\|f X_{j} \eta_{R}\right\|_{X_{\alpha}^{p, q}}<\varepsilon / 2, j=1, \ldots, \ell$. Therefore,

$$
\left\|f-f \eta_{R}\right\|_{X_{\alpha}^{p, q}}<\varepsilon, \quad \text { and } \quad\left\|X_{j} f-X_{j}\left(f \eta_{R}\right)\right\|_{X_{\alpha}^{p, q}}<\varepsilon,
$$

that is, $\left\|f-f \eta_{R}\right\|_{X_{\alpha+1}^{p, q}} \lesssim \varepsilon$. 
Next, given $f \in X_{\alpha+1}^{p, q}$ having compact support, let $\left\{\psi_{\kappa}\right\}, 0<\kappa \leq 1 / 2$ be the approximation of the identity of Step 3, and consider $\psi_{\kappa} * f$. Then, by Steps 4 and $5, \psi_{\kappa} * f \rightarrow f, X_{j}\left(\psi_{\kappa} * f\right)=\psi_{\kappa} * X_{j} f \rightarrow X_{j} f, j=1, \ldots, \ell$, in $X_{\alpha}^{p, q}$, as $\kappa \rightarrow 0$. This implies that $\psi_{\kappa} f \rightarrow f$ in $X_{\alpha+1}^{p, q}$, as $\kappa \rightarrow 0$. This shows that $C_{c}^{\infty}$ is dense in $X_{\alpha+1}^{p, q}$, for $\alpha \in(0,1)$.

Step 7 We now finish the proof. Arguing as in the previous step, we obtain that $C_{c}^{\infty}$ is dense in $X_{\alpha}^{p, q}$ for all $\alpha \in(0, \infty) \backslash \mathbb{N}$. Let $n$ be a positive integer, and $\theta \in(0,1)$. By [3, Theorem 4.2.2] $X_{n+\theta}^{p, q}=X_{n-\theta}^{p, q} \cap X_{n+\theta}^{p, q}$ is dense in $X_{n}^{p, q}$. Since $C_{c}^{\infty}$ is dense in $X_{n+\theta}^{p, q}$ which embeds continuously into $X_{n}^{p, q}$, we deduce that $C_{c}^{\infty}$ is dense in $X_{n}^{p, q}$.

\section{Isomorphisms of Triebel-Lizorkin and Besov Spaces}

Goal of this section is to prove that Bessel potentials provide isomorphisms in both the Triebel-Lizorkin and Besov scales and that a simplified version of local Riesz transforms is bounded on both the Triebel-Lizorkin and Besov spaces. We continue to denote by $X_{\alpha}^{p, q}$ either $F_{\alpha}^{p, q}$ or $B_{\alpha}^{p, q}$. We begin by showing that for all $c \geq 0$, the fractional powers of $\Delta_{\chi}+c I$ are bounded on the spaces $X_{\alpha}^{p, q}$. Precisely, we prove the following.

Lemma 12 Let $p, q \in(1,+\infty), \alpha \geq 0$ and let $\gamma>0$. Then, for all $c \geq 0$,

$$
\left(\Delta_{\chi}+c I\right)^{\gamma / 2}: X_{\alpha+\gamma}^{p, q} \rightarrow X_{\alpha}^{p, q}
$$

is bounded.

Proof If $\beta>0$, then for $\tau>0$ we have

$$
\tau^{-\beta}=\frac{1}{\Gamma(\beta)} \int_{0}^{+\infty} s^{\beta-1} e^{-\tau s} d s,
$$

so that, if $\gamma>0, c \geq 0$ and $k$ is an integer, $k \geq[\gamma / 2]+1$, for every $f \in \mathcal{S}$

$$
\begin{aligned}
\left(\Delta_{\chi}+c I\right)^{\gamma / 2} f & =\left(\Delta_{\chi}+c I\right)^{-(k-\gamma / 2)}\left(\Delta_{\chi}+c I\right)^{k} f \\
& =\frac{1}{\Gamma(k-\gamma / 2)} \int_{0}^{+\infty} s^{k-\gamma / 2} e^{-s\left(\Delta_{\chi}+c I\right)}\left(\Delta_{\chi}+c I\right)^{k} f \frac{d s}{s} \\
& =\frac{1}{\Gamma(k-\gamma / 2)} \sum_{j=0}^{k} \sigma_{j} \int_{0}^{+\infty} s^{k-\gamma / 2} e^{-c s} e^{-s \Delta_{\chi}} \Delta_{\chi}^{j} f \frac{d s}{s}
\end{aligned}
$$

for suitable positive constants $\sigma_{j}$. 
Step 1 We prove that for all $f \in X_{\alpha}^{p, q}$

$$
\left\|e^{-\frac{1}{2} \Delta_{\chi}}\left(\Delta_{\chi}+c I\right)^{\gamma / 2} f\right\|_{L^{p}\left(\mu_{\chi}\right)} \lesssim\left\|e^{-\frac{1}{4} \Delta_{\chi}} f\right\|_{L^{p}\left(\mu_{\chi}\right)} .
$$

Using (48), [6, Lemma 3.3], the Cauchy-Schwarz inequality and the boundedness of the $g$-function (9), we notice that

$$
\begin{aligned}
& \left\|e^{-\frac{1}{2} \Delta_{\chi}}\left(\Delta_{\chi}+c I\right)^{\gamma / 2} f\right\|_{L^{p}\left(\mu_{\chi}\right)} \\
& \lesssim \sum_{j=0}^{k} \int_{0}^{3 / 4} s^{k-\gamma / 2}\left\|e^{-(s+1 / 4) \Delta_{\chi}} \Delta_{\chi}^{j} e^{-\frac{1}{4} \Delta_{\chi}} f\right\|_{L^{p}\left(\mu_{\chi}\right)} \frac{d s}{s}+\left\|e^{-\frac{1}{4} \Delta_{\chi}} f\right\|_{L^{p}\left(\mu_{\chi}\right)} \\
& +\sum_{j=1}^{k}\left\|\left(\int_{3 / 4}^{+\infty}\left|\left(s \Delta_{\chi}\right)^{j} e^{-s \Delta_{\chi}} e^{-\frac{1}{2} \Delta_{\chi}} f\right|^{2} \frac{d s}{s}\right)^{1 / 2}\right\|_{L^{p}\left(\mu_{\chi}\right)} \\
& \lesssim \sum_{j=0}^{k} \int_{0}^{1} \frac{s^{k-\gamma / 2}}{(s+1 / 4)^{j}}\left\|e^{-\frac{1}{4} \Delta_{\chi}} f\right\|_{L^{p}\left(\mu_{\chi}\right)} \frac{d s}{s}+\left\|e^{-\frac{1}{4} \Delta_{\chi}} f\right\|_{L^{p}\left(\mu_{\chi}\right)} \\
& +\sum_{j=1}^{k}\left\|g_{j}\left(e^{-\frac{1}{2} \Delta_{\chi}} f\right)\right\|_{L^{p}\left(\mu_{\chi}\right)} \\
& \lesssim\left\|e^{-\frac{1}{4} \Delta_{\chi}} f\right\|_{L^{p}\left(\mu_{\chi}\right)},
\end{aligned}
$$

and thus (49) holds true.

In order to proceed with the main part of the estimates, we need to consider, for $m>\alpha / 2$,

$$
\begin{aligned}
& W_{t}^{(m)}\left(\Delta_{\chi}+c I\right)^{\gamma / 2} f \\
& =\frac{1}{\Gamma(k-\gamma / 2)} \sum_{j=0}^{k} \sigma_{j}\left(\int_{0}^{1}+\int_{1}^{+\infty}\right) s^{k-\gamma / 2} e^{-s \Delta_{\chi}} e^{-c s} \Delta_{\chi}^{j} W_{t}^{(m)} f \frac{d s}{s} \\
& =: \sum_{j=0}^{k} F_{1}^{j}(t, \cdot)+F_{\infty}^{j}(t, \cdot) .
\end{aligned}
$$

Observe that $F_{1}^{j}, F_{\infty}^{j}$ are functions defined on $(0,1) \times G$.

Step 2 We prove that

$$
\left(\Delta_{\chi}+c I\right)^{\gamma / 2}: B_{\alpha+\gamma}^{p, q} \rightarrow B_{\alpha}^{p, q}
$$


is bounded, by showing that for any $f \in \mathcal{S}$

$$
\begin{aligned}
& \int_{0}^{1}\left(t^{-\alpha / 2}\left\|W_{t}^{(m)}\left(\Delta_{\chi}+c I\right)^{\gamma / 2} f\right\|_{L^{p}\left(\mu_{\chi}\right)}\right)^{q} \frac{d t}{t} \\
& \lesssim \int_{0}^{1}\left(t^{-(\alpha+\gamma) / 2}\left\|W_{t}^{(m+k)} f\right\|_{L^{p}\left(\mu_{\chi}\right)}\right)^{q} \frac{d t}{t} .
\end{aligned}
$$

By the norm equivalence in Theorem 6 and (49), Step 2 will follow.

We prove (51) using the decomposition (50). First, observe that

$$
\begin{aligned}
\| F_{1}^{0}(t, \cdot) & +F_{\infty}^{0}(t, \cdot) \|_{L^{p}\left(\mu_{\chi}\right)} \\
& \lesssim\left\|\int_{0}^{+\infty} s^{k-\gamma / 2} e^{-c s}\left|e^{-s \Delta_{\chi}} W_{t}^{(m)} f\right| \frac{d s}{s}\right\|_{L^{p}\left(\mu_{\chi}\right)} \lesssim\left\|W_{t}^{(m)} f\right\|_{L^{p}\left(\mu_{\chi}\right)} .
\end{aligned}
$$

Thus, we may suppose $j \geq 1$. By the Cauchy-Schwarz inequality and the boundedness of the $g$-function (9)

$$
\begin{aligned}
\left\|F_{\infty}^{j}(t, \cdot)\right\|_{L^{p}\left(\mu_{\chi}\right)} & \lesssim\left\|\int_{1}^{+\infty} s^{k-\gamma / 2} e^{-c s}\left|e^{-s \Delta_{\chi}} \Delta_{\chi}^{j} W_{t}^{(m)} f\right| \frac{d s}{s}\right\|_{L^{p}\left(\mu_{\chi}\right)} \\
& \lesssim\left\|\left(\int_{1}^{+\infty}\left|\left(s \Delta_{\chi}\right)^{j} e^{-s \Delta_{\chi}}\left(W_{t}^{(m)} f\right)\right|^{2} \frac{d s}{s}\right)^{1 / 2}\right\|_{L^{p}\left(\mu_{\chi}\right)} \\
& \lesssim\left\|g_{j}\left(W_{t}^{(m)} f\right)\right\|_{L^{p}\left(\mu_{\chi}\right)} \\
& \lesssim\left\|W_{t}^{(m)} f\right\|_{L^{p}\left(\mu_{\chi}\right)} .
\end{aligned}
$$

Therefore,

$$
\begin{aligned}
\int_{0}^{1}\left(t^{-\alpha / 2}\left\|F_{\infty}^{j}(t, \cdot)\right\|_{L^{p}\left(\mu_{\chi}\right)}\right)^{q} \frac{d t}{t} \\
\quad \lesssim \int_{0}^{1}\left(t^{-\alpha / 2}\left\|W_{t}^{(m)} f\right\|_{L^{p}\left(\mu_{\chi}\right)}\right)^{q} \frac{d t}{t} \lesssim\|f\|_{B_{\alpha}^{p, q}}^{q} \lesssim\|f\|_{B_{\alpha+\gamma}^{p, q}}^{q}
\end{aligned}
$$

Next,

$$
\begin{aligned}
\left\|F_{1}^{j}(t, \cdot)\right\|_{L^{p}\left(\mu_{\chi}\right)} & \lesssim \int_{0}^{1} s^{k-\gamma / 2}\left\|e^{-s \Delta_{\chi}} \Delta_{\chi}^{j} W_{t}^{(m)} f\right\|_{L^{p}\left(\mu_{\chi}\right)} \frac{d s}{s} \\
& =\left(\int_{0}^{t}+\int_{t}^{1}\right) t^{-j} s^{k-\gamma / 2}\left\|e^{-s \Delta_{\chi}} W_{t}^{(m+j)} f\right\|_{L^{p}\left(\mu_{\chi}\right)} \frac{d s}{s} \\
& =: I_{j}(t)+I I_{j}(t) .
\end{aligned}
$$


Now,

$$
\begin{aligned}
I_{j}(t) & =\int_{0}^{t} t^{-j} s^{k-\gamma / 2}\left\|e^{-s \Delta_{\chi}} W_{t}^{(m+j)} f\right\|_{L^{p}\left(\mu_{\chi}\right)} \frac{d s}{s} \\
& \lesssim t^{-j}\left\|W_{t}^{(m+j)} f\right\|_{L^{p}\left(\mu_{\chi}\right)} \int_{0}^{t} s^{k-1-\gamma / 2} d s \\
& \approx t^{k-j-\gamma / 2}\left\|W_{t}^{(m+j)} f\right\|_{L^{p}\left(\mu_{\chi}\right)},
\end{aligned}
$$

so that, since $m+j>(\alpha+\gamma+2 j-2 k) / 2$,

$$
\begin{aligned}
\int_{0}^{1}\left(t^{-\alpha / 2} I_{j}(t)\right)^{q} \frac{d t}{t} \lesssim \int_{0}^{1}\left(t^{-(\alpha+\gamma+2 j-2 k) / 2}\left\|W_{t}^{(m+j)} f\right\|_{L^{p}\left(\mu_{\chi}\right)}\right)^{q} \frac{d t}{t} \\
\lesssim\|f\|_{B_{\alpha+\gamma+2 j-2 k}^{p, q}}^{q} \lesssim\|f\|_{B_{\alpha+\gamma}^{p, q}}^{q} .
\end{aligned}
$$

Next,

$$
\begin{aligned}
t^{-\alpha / 2} I I_{j}(t) & =\int_{t}^{1} t^{-j-\alpha / 2} s^{k-\gamma / 2}\left\|e^{-s \Delta_{\chi}} W_{t}^{(m+j)} f\right\|_{L^{p}\left(\mu_{\chi}\right)} \frac{d s}{s} \\
& \leq \int_{0}^{1} \mathbf{1}_{\{t<s\}}\left(\frac{t}{s}\right)^{m-\alpha / 2} s^{-(\alpha+\gamma+2 j-2 k) / 2}\left\|W_{s}^{(m+j)} f\right\|_{L^{p}\left(\mu_{\chi}\right)} \frac{d s}{s} .
\end{aligned}
$$

Hence,

$$
\int_{0}^{1}\left(t^{-\alpha / 2} I I_{j}(t)\right)^{q} \frac{d t}{t} \leq \int_{0}^{1}\left(\int_{0}^{1} K(s, t) g(s) \frac{d s}{s}\right)^{q} \frac{d t}{t}
$$

where

$$
K(s, t)=\mathbf{1}_{\{t<s\}}\left(\frac{t}{s}\right)^{m-\alpha / 2} \quad \text { and } \quad g(s)=s^{-(\alpha+\gamma+2 j-2 k) / 2}\left\|W_{s}^{(m+j)} f\right\|_{L^{p}\left(\mu_{\chi}\right)} .
$$

It is easy to check that

$$
\int_{0}^{1} K(s, t) \frac{d s}{s} \lesssim 1 \text { and } \int_{0}^{1} K(s, t) \frac{d t}{t} \lesssim 1
$$

so that Schur's lemma (see [11] e.g.) gives that

$$
\int_{0}^{1}\left(t^{-\alpha / 2} I I_{j}(t)\right)^{q} \frac{d t}{t} \lesssim \int_{0}^{1}|g(t)|^{q} \frac{d t}{t} .
$$

Thus, putting together (52) to (55) we obtain (51). This completes Step 2. 
Potential Spaces on Lie Groups

183

Step 3 We now prove that

$$
\left(\Delta_{\chi}+c I\right)^{\gamma / 2}: F_{\alpha+\gamma}^{p, q} \rightarrow F_{\alpha}^{p, q}
$$

is bounded, by showing that for any $f \in \mathcal{S}$

$$
\begin{aligned}
\|\left(\int _ { 0 } ^ { 1 } \left(t^{-\alpha / 2} \mid W_{t}^{(m)}\right.\right. & \left.\left.\left(\Delta_{\chi}+c I\right)^{\gamma / 2} f \mid\right)^{q} \frac{d t}{t}\right)^{1 / q} \|_{L^{p}\left(\mu_{\chi}\right)} \\
& \lesssim\left\|\left(\int_{0}^{1}\left(t^{-(\alpha+\gamma) / 2}\left|W_{t}^{(m+k)} f\right|\right)^{q} \frac{d t}{t}\right)^{1 / q}\right\|_{L^{p}\left(\mu_{\chi}\right)} .
\end{aligned}
$$

Again, this, together with Theorem 6 and (49), will give the desired boundedness.

We use decomposition (50) again.

$$
\begin{aligned}
F_{\infty}^{j}(t, \cdot) & =\frac{\sigma_{j}}{\Gamma(k-\gamma / 2)} t^{m} e^{-t \Delta_{\chi}} \int_{1}^{+\infty} s^{k-\gamma / 2} e^{-c s} e^{-(s-1 / 2) \Delta_{\chi}} \Delta_{\chi}^{m+j} e^{-\frac{1}{2} \Delta_{\chi}} f \frac{d s}{s} \\
& =\frac{\sigma_{j}}{\Gamma(k-\gamma / 2)} t^{m} e^{-t \Delta_{\chi}} \int_{\frac{1}{2}}^{+\infty}\left(s+\frac{1}{2}\right)^{k-1-\gamma / 2} e^{-c\left(s+\frac{1}{2}\right)} e^{-s \Delta_{\chi}} \Delta_{\chi}^{m+j} e^{-\frac{1}{2} \Delta_{\chi}} f d s .
\end{aligned}
$$

We notice that, since $f \in \mathcal{S}$ and $e^{-t \Delta_{\chi}}$ is continuous on $\mathcal{S}^{\prime}$, the integral converges in $\mathcal{S}^{\prime}$. By the Cauchy-Schwarz inequality and recalling (8), we have

$$
\begin{aligned}
\left|F_{\infty}^{j}(t, \cdot)\right| & \lesssim t^{m} e^{-t \Delta_{\chi}}\left(\int_{0}^{+\infty}\left|\left(s \Delta_{\chi}\right)^{m+j} e^{-s \Delta_{\chi}} e^{-\frac{1}{2} \Delta_{\chi}} f\right|^{2} \frac{d s}{s}\right)^{1 / 2} \\
& =t^{m} e^{-t \Delta_{\chi}} g_{m+j}\left(e^{-\frac{1}{2} \Delta_{\chi}} f\right) .
\end{aligned}
$$

Now we use [6, Proposition 3.6 (ii)] and the boundedness of the $g$-function to obtain

$$
\begin{aligned}
& \left\|\left(\int_{0}^{1}\left(t^{-\alpha / 2}\left|F_{\infty}^{j}(t, \cdot)\right|\right)^{q} \frac{d t}{t}\right)^{1 / q}\right\|_{L^{p}\left(\mu_{\chi}\right)} \\
& \lesssim\left\|\left(\int_{0}^{1}\left(t^{m-\alpha / 2} e^{-t \Delta_{\chi}} g_{m+j}\left(e^{-\frac{1}{2} \Delta_{\chi}} f\right)\right)^{q} \frac{d t}{t}\right)^{1 / q}\right\|_{L^{p}\left(\mu_{\chi}\right)} \\
& \lesssim\left\|g_{m+j}\left(e^{-\frac{1}{2} \Delta_{\chi}} f\right)\right\|_{L^{p}\left(\mu_{\chi}\right)} \\
& \lesssim\left\|e^{-\frac{1}{2} \Delta_{\chi}} f\right\|_{L^{p}\left(\mu_{\chi}\right)} .
\end{aligned}
$$


Finally,

$$
\begin{aligned}
& \int_{0}^{1}\left(t^{-\alpha / 2} F_{1}^{j}(t, \cdot)\right)^{q} \frac{d t}{t} \\
& \lesssim \int_{0}^{1}\left(t^{-\alpha / 2}\left(\int_{0}^{t}+\int_{t}^{1}\right) s^{k-\gamma / 2}\left|e^{-s \Delta_{\chi}} \Delta_{\chi}^{j} W_{t}^{(m)} f\right| \frac{d s}{s}\right)^{q} \frac{d t}{t} \\
& \lesssim \int_{0}^{1}\left(t^{-\alpha / 2} \int_{0}^{t} s^{k-\gamma / 2}\left|e^{-s \Delta_{\chi}} \Delta_{\chi}^{j} W_{t}^{(m)} f\right| \frac{d s}{s}\right)^{q} \frac{d t}{t} \\
& \quad+\int_{0}^{1}\left(t^{-\alpha / 2} \int_{t}^{1} s^{k-\gamma / 2}\left|e^{-s \Delta_{\chi}} \Delta_{\chi}^{j} W_{t}^{(m)} f\right| \frac{d s}{s}\right)^{q} \frac{d t}{t} \\
& =: I_{j}+I I_{j},
\end{aligned}
$$

where in this case, $I_{j}$ and $I I_{j}$ are functions on $G$. Similarly to the argument in Step 1 , we have

$$
\begin{aligned}
I_{j} & =\int_{0}^{1}\left(\int_{0}^{t} \frac{t^{m-\alpha / 2} s^{k-\gamma / 2}}{(s+t)^{m+j}}\left|W_{s+t}^{(m+j)} f\right| \frac{d s}{s}\right)^{q} \frac{d t}{t} \\
& =\int_{0}^{1}\left(\int_{t}^{2 t} \frac{t^{m-\alpha / 2}(\tau-t)^{k-1-\gamma / 2}}{\tau^{m+j-1}}\left|W_{\tau}^{(m+j)} f\right| \frac{d \tau}{\tau}\right)^{q} \frac{d t}{t} \\
& =\int_{0}^{1}\left(\int_{0}^{1} K(\tau, t) g(\tau) \frac{d \tau}{\tau}\right)^{q} \frac{d t}{t}
\end{aligned}
$$

where

$$
K(\tau, t)=\mathbf{1}_{\{t<\tau<2 t\}} \frac{t^{m-\alpha / 2}(\tau-t)^{k-1-\gamma / 2}}{\tau^{m+k-1-(\alpha+\gamma) / 2}} \text { and } g(\tau)=\tau^{-(\alpha+\gamma+2 j-2 k) / 2}\left|W_{\tau}^{(m+k)} f\right| .
$$

Since

$$
\int_{0}^{1} K(\tau, t) \frac{d \tau}{\tau} \lesssim 1 \text { and } \int_{0}^{1} K(\tau, t) \frac{d t}{t} \lesssim 1
$$

we obtain that

$$
I_{j} \lesssim \int_{0}^{1}\left(t^{-(\alpha+\gamma+2 j-2 k) / 2}\left|W_{t}^{(m+k)} f\right|\right)^{q} \frac{d t}{t}
$$


To estimate $I I_{j}$ we use [6, Proposition 3.6 (iv)] and obtain that

$$
\begin{aligned}
& \left\|I I_{j}^{1 / q}\right\|_{L^{p}\left(\mu_{\chi}\right)} \\
& =\left\|\left(\int_{0}^{1}\left(t^{m-\alpha / 2} \int_{t}^{1} s^{-m+k-j-\gamma / 2}\left|e^{-t \Delta_{\chi}} W_{s}^{(m+j)} f\right| \frac{d s}{s}\right)^{q} \frac{d t}{t}\right)^{1 / q}\right\|_{L^{p}\left(\mu_{\chi}\right)} \\
& \leq\left\|\left(\int_{0}^{1}\left(t^{m-\alpha / 2} e^{-t \Delta_{\chi}} \int_{t}^{1} s^{-m+k-j-\gamma / 2}\left|W_{s}^{(m+j)} f\right| \frac{d s}{s}\right)^{q} \frac{d t}{t}\right)^{1 / q}\right\|_{L^{p}\left(\mu_{\chi}\right)} \\
& \lesssim\left\|\left(\int_{0}^{1}\left(t^{m-\alpha / 2} \int_{t}^{1} s^{-m+k-j-\gamma / 2}\left|W_{s}^{(m+j)} f\right| \frac{d s}{s}\right)^{q} \frac{d t}{t}\right)^{1 / q}\right\|_{L^{p}\left(\mu_{\chi}\right)} \\
& =:\left\|\left(\int_{0}^{1}\left(\int_{0}^{1} K(s, t) g(s) \frac{d s}{s}\right)^{q} \frac{d t}{t}\right)^{1 / q}\right\|_{L^{p}\left(\mu_{\chi}\right)},
\end{aligned}
$$

where we have set

$$
K(s, t)=\mathbf{1}_{\{t<s\}}\left(\frac{t}{s}\right)^{m-\alpha / 2} \quad \text { and } \quad g(s)=s^{-(\alpha+\gamma+2 j-2 k) / 2}\left|W_{s}^{(m+j)} f\right| .
$$

Again, since

$$
\int_{0}^{1} K(s, t) \frac{d s}{s} \lesssim 1 \text { and } \int_{0}^{1} K(s, t) \frac{d t}{t} \lesssim 1
$$

we see that

$$
\left\|I I_{j}^{1 / q}\right\|_{L^{p}\left(\mu_{\chi}\right)} \lesssim\left\|\left(\int_{0}^{1}\left(t^{-(\alpha+\gamma+2 j-2 k) / 2}\left|W_{t}^{(m+j)} f\right|\right)^{q} \frac{d t}{t}\right)^{1 / q}\right\|_{L^{p}\left(\mu_{\chi}\right)} .
$$

Therefore, (58), (59) and (61) give

$$
\begin{aligned}
& \left\|\left(\int_{0}^{1}\left(t^{-\alpha / 2} F_{1}^{j}(t, \cdot)\right)^{q} \frac{d t}{t}\right)^{1 / q}\right\|_{L^{p}\left(\mu_{\chi}\right)} \\
& \lesssim\left\|I_{j}^{1 / q}\right\|_{L^{p}\left(\mu_{\chi}\right)}+\left\|I I_{j}^{1 / q}\right\|_{L^{p}\left(\mu_{\chi}\right)} \\
& \lesssim\left\|\left(\int_{0}^{1}\left(t^{-(\alpha+\gamma+2 j-2 k) / 2}\left|W_{t}^{(m+j)} f\right|\right)^{q} \frac{d t}{t}\right)^{1 / q}\right\|_{L^{p}\left(\mu_{\chi}\right)} \\
& \lesssim\|f\|_{F_{\alpha+\gamma+2 j-2 k}^{p, q}} \lesssim\|f\|_{F_{\alpha+\gamma}^{p, q} .} .
\end{aligned}
$$


This, together with (57), proves (56), and finally estimates (49) and (56) complete Step 3. The proof of the lemma is complete.

We are finally ready to prove the main result of this section.

Theorem 13 Let $\alpha \geq 0, \gamma \geq 0, p, q \in(1,+\infty)$, and let $X_{\alpha}^{p, q}$ denote either space $F_{\alpha}^{p, q}\left(\mu_{\chi}\right)$ or $B_{\alpha}^{p, q}\left(\mu_{\chi}\right)$. Then, for $c>0$ sufficiently large,

$$
\left(\Delta_{\chi}+c I\right)^{\gamma / 2}: X_{\alpha+\gamma}^{p, q} \rightarrow X_{\alpha}^{p, q}
$$

is a surjective isomorphism, and its inverse is $\left(\Delta_{\chi}+c I\right)^{-\gamma / 2}$. Moreover, if $c>0$ is sufficiently large, then, for all $\alpha, \gamma \geq 0$, the operators

$$
\Delta_{\chi}^{\gamma}\left(\Delta_{\chi}+c I\right)^{-\gamma}: X_{\alpha}^{p, q} \rightarrow X_{\alpha}^{p, q}
$$

are bounded.

We point out that, for any $\gamma>0, c$ is to be chosen so that the local Riesz transforms $X_{J}\left(\Delta_{\chi}+c I\right)^{-|J| / 2}$ are bounded on $L^{p}\left(\mu_{\chi}\right)$, for $1<p<\infty$ and $|J| \leq[\gamma / 2]+1$. Moreover, the operators $\Delta_{\chi}^{\gamma}\left(\Delta_{\chi}+c I\right)^{-\gamma}$ can be thought as a simplified version of the local Riesz transforms.

Proof Step 1 We first prove that, for $n \in \mathbb{N}$, and $c>0$ sufficiently large so that the local Riesz transforms $X_{J}\left(\Delta_{\chi}+c I\right)^{-n}$ are bounded on $L^{p}\left(\mu_{\chi}\right)$, for $1<p<\infty$ and $|J| \leq 2 n$,

$$
\left(\Delta_{\chi}+c I\right)^{-n}: X_{\alpha}^{p, q} \rightarrow X_{\alpha+2 n}^{p, q}
$$

is bounded.

Since $\left(\Delta_{\chi}+c I\right)^{-\beta}: L^{p}\left(\mu_{\chi}\right) \rightarrow L^{p}\left(\mu_{\chi}\right)$ is bounded, for $p \in(1,+\infty)$ and $\beta>0$, see [29] or also [5], we trivially have that

$$
\left\|e^{-\frac{1}{2} \Delta_{\chi}}\left(\Delta_{\chi}+c I\right)^{-n} f\right\|_{L^{p}\left(\mu_{\chi}\right)} \lesssim\left\|e^{-\frac{1}{2} \Delta_{\chi}} f\right\|_{L^{p}\left(\mu_{\chi}\right)} .
$$

Let $m \geq[\alpha / 2]+1$. In the case of Besov spaces, it suffices to apply Theorem 3.2 in [5] to obtain

$$
\begin{aligned}
\left\|W_{t}^{(m+n)}\left(\Delta_{\chi}+c I\right)^{-n} f\right\|_{L^{p}\left(\mu_{\chi}\right)} & =\left\|\left(t \Delta_{\chi}\right)^{n}\left(\Delta_{\chi}+c I\right)^{-n} W_{t}^{(m)} f\right\|_{L^{p}\left(\mu_{\chi}\right)} \\
& \lesssim t^{n} \sum_{|J| \leq 2 n}\left\|X_{J}\left(\Delta_{\chi}+c I\right)^{-n} W_{t}^{(m)} f\right\|_{L^{p}\left(\mu_{\chi}\right)} \\
& \lesssim t^{n}\left\|W_{t}^{(m)} f\right\|_{L^{p}\left(\mu_{\chi}\right)} .
\end{aligned}
$$


Hence,

$$
\begin{aligned}
& \left(\int_{0}^{1}\left(t^{-(n+\alpha / 2)}\left\|W_{t}^{(m+n)}\left(\Delta_{\chi}+c I\right)^{-n} f\right\|_{L^{p}\left(\mu_{\chi}\right)}\right)^{q} \frac{d t}{t}\right)^{1 / q} \\
& \lesssim\left(\int_{0}^{1}\left(t^{-\alpha / 2}\left\|W_{t}^{(m)} f\right\|_{L^{p}\left(\mu_{\chi}\right)}\right)^{q} \frac{d t}{t}\right)^{1 / q} \\
& \lesssim\|f\|_{B_{\alpha}^{p, q}} .
\end{aligned}
$$

Therefore, (63) and (64) show that $\left(\Delta_{\chi}+c I\right)^{-n}: B_{\alpha}^{p, q} \rightarrow B_{\alpha+2 n}^{p, q}$ is bounded, $p, q \in(1,+\infty), \alpha \geq 0, n \in \mathbb{N}$.

Next we consider the case of the Triebel-Lizorkin spaces. Arguing as in (48) we write

$$
\begin{aligned}
W_{t}^{(m+n)}\left(\Delta_{\chi}+c I\right)^{-n} f & =\frac{1}{\Gamma(n)} \int_{0}^{+\infty} s^{n} e^{-c s} e^{-s \Delta_{\chi}} W_{t}^{(m+n)} f \frac{d s}{s} \\
& =\frac{1}{\Gamma(n)}\left(\int_{0}^{1}+\int_{1}^{+\infty}\right) s^{n} e^{-c s} e^{-s \Delta_{\chi}} W_{t}^{(m+n)} f \frac{d s}{s} \\
& =: \frac{1}{\Gamma(n)}\left(A_{1}(t, \cdot)+A_{\infty}(t, \cdot)\right) .
\end{aligned}
$$

We begin with the latter term and observe that, since $f \in \mathcal{S}$ and $e^{-t \Delta_{\chi}}$ is continuous on $\mathcal{S}^{\prime}$, we get

$$
\left|A_{\infty}(t, \cdot)\right|=t^{m+n}\left|e^{-t \Delta_{\chi}} \int_{1}^{+\infty} s^{n} e^{-c s} e^{-(s-1 / 2) \Delta_{\chi}} \Delta_{\chi}^{m+n} e^{-\frac{1}{2} \Delta_{\chi}} f \frac{d s}{s}\right|
$$

which by the Cauchy-Schwarz inequality is bounded by

$$
t^{m+n} e^{-t \Delta_{\chi}}\left(\int_{0}^{+\infty}\left|W_{s}^{(m+n)} e^{-\frac{1}{2} \Delta_{\chi}} f\right|^{2} \frac{d s}{s}\right)^{1 / 2}=t^{m+n} e^{-t \Delta_{\chi}} g_{m+n}\left(e^{-\frac{1}{2} \Delta_{\chi}} f\right),
$$

where $g_{m+n}$ is defined in (8). Therefore, by the above estimate, Proposition 3.6 (ii) in [6] and the boundedness of the $g$-function,

$$
\begin{aligned}
& \left\|\left(\int_{0}^{1}\left(t^{-(n+\alpha / 2)}\left|A_{\infty}(t, \cdot)\right|\right)^{q} \frac{d t}{t}\right)^{1 / q}\right\|_{L^{p}\left(\mu_{\chi}\right)} \\
& \lesssim\left\|\left(\int_{0}^{1}\left(t^{m-\alpha / 2} e^{-t \Delta_{\chi}} g_{m+n}\left(e^{-\frac{1}{2} \Delta_{\chi}} f\right)\right)^{q} \frac{d t}{t}\right)^{1 / q}\right\|_{L^{p}\left(\mu_{\chi}\right)} \\
& \lesssim\left\|g_{m+n}\left(e^{-\frac{1}{2} \Delta_{\chi}} f\right)\right\|_{L^{p}\left(\mu_{\chi}\right)} \\
& \lesssim\left\|e^{-\frac{1}{2} \Delta_{\chi}} f\right\|_{L^{p}\left(\mu_{\chi}\right)} .
\end{aligned}
$$


Now we turn to $A_{1}(t, \cdot)$, and arguing as above and applying [6, Lemma 3.2 (i)] we get

$$
\begin{aligned}
& \left|A_{1}(t, \cdot)\right| \\
& \leq t^{m+n}\left(\int_{0}^{t} s^{n}\left|e^{-\left(s+\frac{t}{2}\right) \Delta_{\chi}} e^{-\frac{t}{2} \Delta_{\chi}} \Delta_{\chi}^{m+n} f\right| \frac{d s}{s}+e^{-t \Delta_{\chi}} \int_{t}^{1} s^{n}\left|e^{-s \Delta_{\chi}} \Delta_{\chi}^{m+n} f\right| \frac{d s}{s}\right) \\
& \lesssim t^{m+n}\left(e^{-c t \Delta_{\chi}} \int_{0}^{t} s^{n}\left|e^{-\frac{t}{2} \Delta_{\chi}} \Delta_{\chi}^{m+n} f\right| \frac{d s}{s}+e^{-t \Delta_{\chi}} \int_{t}^{1} s^{n}\left|e^{-s \Delta_{\chi}} \Delta_{\chi}^{m+n} f\right| \frac{d s}{s}\right) \\
& =t^{m+n}\left(t^{n} e^{-c t \Delta_{\chi}}\left|e^{-\frac{t}{2} \Delta_{\chi}} \Delta_{\chi}^{m+n} f\right|+e^{-t \Delta_{\chi}} \int_{t}^{1} s^{n}\left|e^{-s \Delta_{\chi}} \Delta_{\chi}^{m+n} f\right| \frac{d s}{s}\right) .
\end{aligned}
$$

As for the first term, by [6, Proposition 3.6 (i)] one has

$$
\begin{aligned}
& \left\|\left(\int_{0}^{1}\left(t^{-\left(n+\frac{\alpha}{2}\right)} t^{m+2 n} e^{-c t \Delta_{\chi}}\left|e^{-\frac{t}{2} \Delta_{\chi}} \Delta_{\chi}^{m+n} f\right|\right)^{q} \frac{d t}{t}\right)^{1 / q}\right\|_{L^{p}\left(\mu_{\chi}\right)} \\
& \lesssim\left\|\left(\int_{0}^{1} t^{-q \frac{\alpha}{2}}\left|W_{t}^{(m+n)} f\right|^{q} \frac{d t}{t}\right)^{1 / q}\right\|_{L^{p}\left(\mu_{\chi}\right)} \\
& \lesssim\|f\|_{F_{\alpha}^{p, q}} .
\end{aligned}
$$

For the second term on the right hand side of (66) we use Schur's test. Precisely, arguing as at the end of Step 2 in the proof of Lemma 12, we have

$$
\begin{aligned}
& \int_{0}^{1}\left(t^{m-\alpha / 2} \int_{t}^{1} s^{n}\left|e^{-s \Delta_{\chi}} \Delta_{\chi}^{m+n} f\right| \frac{d s}{s}\right)^{q} \frac{d t}{t} \\
& =\int_{0}^{1}\left(\int_{0}^{1} \mathbf{1}_{\{t<s\}}\left(\frac{t}{s}\right)^{m} t^{-\alpha / 2}\left|W_{s}^{(m+n)} f\right| \frac{d s}{s}\right)^{q} \frac{d t}{t} \\
& \lesssim \int_{0}^{1}\left(t^{-\alpha / 2}\left|W_{t}^{(n+m)} f\right|\right)^{q} \frac{d t}{t} .
\end{aligned}
$$

Thus, by (67) and (68) we obtain that

$$
\left\|\left(\int_{0}^{1}\left(t^{-(n+\alpha / 2)}\left|A_{1}(t, \cdot)\right|\right)^{q} \frac{d t}{t}\right)^{1 / q}\right\|_{L^{p}\left(\mu_{\chi}\right)} \lesssim\|f\|_{F_{\alpha}^{p, q} .}
$$

Estimates (65) and (69) complete the proof of Step 1. 
Step 2 We now complete the proof.

We first observe that for $\gamma \geq 0$, the operator

$$
\left(\Delta_{\chi}+c I\right)^{-\gamma / 2}: X_{\alpha}^{p, q} \rightarrow X_{\alpha+\gamma}^{p, q}
$$

is bounded. Indeed, it suffices to notice that, choosing $n \in \mathbb{N}, n>\gamma / 2$ we have

$$
\left(\Delta_{\chi}+c I\right)^{-\gamma / 2}=\left(\Delta_{\chi}+c I\right)^{n-\gamma / 2}\left(\Delta_{\chi}+c I\right)^{-n} .
$$

The conclusion follows from the boundedness of the two operators on the right hand side given by Step 1 and Lemma 12 .

It now follows that

$$
\left(\Delta_{\chi}+c I\right)^{\gamma / 2}: X_{\alpha+\gamma}^{p, q} \rightarrow X_{\alpha}^{p, q}
$$

is a surjective isomorphisms. Indeed, given any $\phi \in \mathcal{S}$, we can write

$$
\phi=\left(\Delta_{\chi}+c I\right)^{\gamma / 2}\left(\Delta_{\chi}+c I\right)^{-\gamma / 2} \phi=\left(\Delta_{\chi}+c I\right)^{-\gamma / 2}\left(\Delta_{\chi}+c I\right)^{\gamma / 2} \phi .
$$

Hence, given any $f \in X_{\alpha}^{p, q}$, we can write

$$
f=\left(\Delta_{\chi}+c I\right)^{\gamma / 2}\left(\Delta_{\chi}+c I\right)^{-\gamma / 2} f,
$$

since $f \in \mathcal{S}^{\prime}$.

Finally, it is now clear that for $\alpha, \gamma \geq 0$,

$$
\Delta_{\chi}^{\gamma / 2}\left(\Delta_{\chi}+c I\right)^{-\gamma / 2}: X_{\alpha}^{p, q} \rightarrow X_{\alpha}^{p, q}
$$

is bounded. The proof is now complete.

Using Theorem 11 and applying the previous theorem with $\alpha=0$ and $\gamma=2 m$, $m$ being a positive integer, we immediately obtain the following

Corollary 14 Let $p, q \in(1, \infty)$ and let $X_{0}^{p, q}$ denote either $F_{0}^{p, q}$ or $B_{0}^{p, q}$. Then, $C_{c}^{\infty}(G)$ is dense in $X_{0}^{p, q}$.

\section{Final Remarks and Open Problems}

In this final section we discuss some directions for future work and indicate some open problems.

First of all, we stress the fact that in this work and [6] we have limited ourselves to the cases $p, q \in(1,+\infty)$ and $\alpha \geq 0$. It would be interesting to investigate whether the spaces $F_{0}^{p, 2}\left(\mu_{\chi}\right)$ with $p=1,+\infty$, correspond respectively to the local Hardy 
space $\mathfrak{h}^{1}\left(\mu_{\chi}\right)$ and its dual $\mathfrak{b m o}\left(\mu_{\chi}\right)$, introduced in [5], in analogy to the Euclidean setting. Such spaces turn out to be useful in many problems, most noticeably in the boundedness of singular integral operators. Moreover, Triebel-Lizorkin and Besov spaces with $0<p<1$ or $0<q<1$ are quasi-Banach and their treatment often requires different techniques. Finally, the spaces $X_{\alpha}^{p, q}\left(\mu_{\chi}\right)$ with $\alpha<0$ should appear as natural duals of the spaces with positive index of regularity and are also of considerable interest.

We recall that Besov and also Triebel-Lizorkin spaces are instrumental to applications to solvability and regularity of solutions of nonlinear differential equations, as, for instance, in the spirit of the results in Section 6 in [5]. It would also be interesting to study the homogeneous versions of Sobolev, Besov and Triebel-Lizorkin spaces in the setting of this work. These spaces, in particular the homogeneous Besov spaces, appear naturally in the Strichartz estimates for the wave equation in the Euclidean space, or Lie groups of polynomial growth, see e.g. [2,19] and [15].

Another set of natural and interesting questions concerns the generalization of some classical geometric inequalities, which have already been studied in the setting of manifolds and metric spaces under suitable geometric assumptions. In particular, we mention the Poincaré inequality, see [31] for the classical case and [27] for Carnot-Carathéodory groups, trace inequalities, see [31] for the classical case and [8] for Carnot-Carathéodory groups, isoperimetric and Sobolev inequalities [31] and [18], to name just a few. We intend to study extensions of these classical inequalities to the case of the sub-Laplacian $\Delta_{\chi}$ on a general Lie group $G$ and of the Sobolev, Triebel-Lizorkin and Besov spaces. We point out that in [45] the authors proved versions of Hardy, Hardy-Sobolev, Caffarelli-Nirenberg, Gagliardo-Nirenberg inequalities in the case of the Sobolev spaces $L_{\alpha}^{p}\left(\mu_{\chi}\right)$.

Acknowledgments We thank Hans Triebel for pointing out some references and sharing with us a preprint version of his new book [54]. We also thank the referee for several corrections and suggestions that greatly improved the presentation of the manuscript.

\section{References}

1. Agrachev, A., Boscain, U., Gauthier, J.-P., Rossi, F.: The intrinsic hypoelliptic Laplacian and its heat kernel on unimodular Lie groups. J. Funct. Anal. 256(8), 2621-2655 (2009)

2. Bahouri, H., Gérard, P., Xu, C.-J.: Espaces de Besov et estimations de Strichartz généralisées sur le groupe de Heisenberg. J. Anal. Math. 82, 93-118 (2000)

3. Bergh, J., Löfström, J.: Interpolation Spaces. An Introduction. Springer-Verlag, Berlin-New York (1976). Grundlehren der Mathematischen Wissenschaften, No. 223

4. Bourbaki, N.: Integration. II. Chapters 7-9. Elements of Mathematics (Berlin). Springer, Berlin (2004). Translated from the 1963 and 1969 French originals by Sterling K. Berberian

5. Bruno, T., Peloso, M.M., Tabacco, A., Vallarino, M.: Sobolev spaces on Lie groups: Embedding theorems and algebra properties. J. Funct.Anal. 276(10), 3014-3050 (2019)

6. Bruno, T., Peloso, M.M., Vallarino, M.: Besov and Triebel-Lizorkin spaces on Lie groups. Math. Ann. 377, 335-377 (2020) 
7. Coulhon, T., Russ, E., Tardivel-Nachef, V.: Sobolev algebras on Lie groups and Riemannian manifolds. Amer. J. Math. 123(2), 283-342 (2001)

8. Danielli, D., Garofalo, N., Nhieu, D.-M.: Trace inequalities for Carnot-Carathéodory spaces and applications. Ann. Scuola Norm. Sup. Pisa Cl. Sci. (4) 27(2), 195-252 (1998)

9. Feneuil, J.: Algebra properties for Besov spaces on unimodular Lie groups. Colloq. Math. 154(2), 205-240 (2018)

10. Folland, G.B.: Subelliptic estimates and function spaces on nilpotent Lie groups. Ark. Mat. 13(2), 161-207 (1975)

11. Folland, G.B.: Real Analysis. Pure and Applied Mathematics (New York), 2nd edn. Wiley, New York (1999). Modern techniques and their applications, A Wiley-Interscience Publication

12. Folland, G.B.: A Course in Abstract Harmonic Analysis, 2nd edn. Textbooks in Mathematics. CRC Press, Boca Raton (2016)

13. Folland, G.B., Stein, E.M.: Estimates for the $\bar{\partial}_{b}$ complex and analysis on the Heisenberg group. Comm. Pure Appl. Math. 27, 429-522 (1974)

14. Furioli, G., Melzi, C., Veneruso, A.: Littlewood-Paley decompositions and Besov spaces on Lie groups of polynomial growth. Math. Nachr. 279(9-10), 1028-1040 (2006)

15. Furioli, G., Melzi, C., Veneruso, A.: Strichartz inequalities for the wave equation with the full Laplacian on the Heisenberg group. Canad. J. Math. 59(6), 1301-1322 (2007)

16. Gallagher, I., Sire, Y.: Besov algebras on Lie groups of polynomial growth. Studia Math. 212(2), 119-139 (2012)

17. García-Cuerva, J., Rubio de Francia, J.L.: Weighted Norm Inequalities and Related Topics. North-Holland Mathematics Studies, vol. 116. North-Holland Publishing Co., Amsterdam (1985). Notas de Matemática [Mathematical Notes], 104

18. Garofalo, N., Nhieu, D.-M.: Isoperimetric and Sobolev inequalities for Carnot-Carathéodory spaces and the existence of minimal surfaces. Commun. Pure Appl. Math. 49(10), 1081-1144 (1996)

19. Ginibre, J., Velo, G.: Generalized Strichartz inequalities for the wave equation. J. Funct. Anal. 133(1), 50-68 (1995)

20. Grafakos, L.: Modern Fourier Analysis. Graduate Texts in Mathematics, 3rd edn., vol. 250. Springer, New York (2014)

21. Guivarc'h, Y.: Croissance polynomiale et périodes des fonctions harmoniques. Bull. Soc. Math. France 101, 333-379 (1973)

22. Han, Y., Müller, D., Yang, D.: A theory of Besov and Triebel-Lizorkin spaces on metric measure spaces modeled on Carnot-Carathéodory spaces. Abstr. Appl. Anal., Art. ID 893409, 250 pp. (2008)

23. Hebisch, W., Mauceri, G., Meda, S.: Spectral multipliers for sub-Laplacians with drift on Lie groups. Math. Z. 251(4), 899-927 (2005)

24. Heikkinen, T., Koskela, P., Tuominen, H.: Approximation and quasicontinuity of Besov and Triebel-Lizorkin functions. Trans. Amer. Math. Soc. 369(5), 3547-3573 (2017)

25. Hörmander, L.: Estimates for translation invariant operators in $L^{p}$ spaces. Acta Math. 104, 93-140 (1960)

26. Hörmander, L.: Hypoelliptic second order differential equations. Acta Math. 119, 147-171 (1967)

27. Jerison, D.: The Poincaré inequality for vector fields satisfying Hörmander's condition. Duke Math. J. 53(2), 503-523 (1986)

28. Kohn, J.J.: Harmonic integrals on strongly pseudo-convex manifolds. I. Ann. Math. (2) 78, $112-148$ (1963)

29. Komatsu, H.: Fractional powers of operators. Pacific J. Math. 19, 285-346 (1966)

30. Korányi, A., Vági, S.: Singular integrals on homogeneous spaces and some problems of classical analysis. Ann. Scuola Norm. Sup. Pisa (3) 25, 575-648 (1971)

31. Leoni, G.: A First Course in Sobolev Spaces. Graduate Studies in Mathematics, 2nd edn., vol. 181. American Mathematical Society, Providence, RI (2017)

32. Martini, A.: Analysis of joint spectral multipliers on Lie groups of polynomial growth. Ann. Inst. Fourier (Grenoble) 62(4), 1215-1263 (2012) 
33. Meda, S.: On the Littlewood-Paley-Stein $g$-function. Trans. Amer. Math. Soc. 347(6), 2201$2212(1995)$

34. Müller, D., Yang, D.: A difference characterization of Besov and Triebel-Lizorkin spaces on RD-spaces. Forum Math. 21(2), 259-298 (2009)

35. Müller, D., Ricci, F., Stein, E.M.: Marcinkiewicz multipliers and multi-parameter structure on Heisenberg (-type) groups. I. Invent. Math. 119(2), 199-233 (1995)

36. Müller, D., Ricci, F., Stein, E.M.: Marcinkiewicz multipliers and multi-parameter structure on Heisenberg (-type) groups. II. Math. Z. 221(2), 267-291 (1996)

37. Müller, D., Peloso, M.M., Ricci, F.: $L^{p}$-spectral multipliers for the Hodge Laplacian acting on 1-forms on the Heisenberg group. Geom. Funct. Anal. 17(3), 852-886 (2007)

38. Müller, D., Peloso, M.M., Ricci, F.: Analysis of the Hodge Laplacian on the Heisenberg group. Mem. Amer. Math. Soc. 233(1095), vi+91 (2015)

39. Peloso, M.M., Ricci, F.: Tangential Cauchy-Riemann equations on quadratic CR manifolds. Atti Accad. Naz. Lincei Cl. Sci. Fis. Mat. Natur. Rend. Lincei (9) Mat. Appl. 13(3-4), 285-294 (2002). Harmonic analysis on complex homogeneous domains and Lie groups (Rome, 2001)

40. Peloso, M.M., Ricci, F.: Analysis of the Kohn Laplacian on quadratic CR manifolds. J. Funct. Anal. 203(2), 321-355 (2003)

41. Peloso, M.M., Vallarino, M.: Sobolev algebras on nonunimodular Lie groups. Calc. Var. Partial Differential Equations 57(6), Art. 150, 34 (2018)

42. Ricci, F., Stein, E.M.: Harmonic analysis on nilpotent groups and singular integrals. I. Oscillatory integrals. J. Funct. Anal. 73(1), 179-194 (1987)

43. Ricci, F., Stein, E.M.: Harmonic analysis on nilpotent groups and singular integrals. II. Singular kernels supported on submanifolds. J. Funct. Anal. 78(1), 56-84 (1988)

44. Ricci, F., Stein, E.M.: Harmonic analysis on nilpotent groups and singular integrals. III. Fractional integration along manifolds. J. Funct. Anal. 86(2), 360-389 (1989)

45. Ruzhansky, M., Yessirkegenov, N.: Hardy, Hardy-Sobolev, Hardy-Littlewood-Sobolev and Caffarelli-Kohn-Nirenberg inequalities on general Lie groups (2018). ArXiv e-prints, $32 \mathrm{pp}$.

46. Stein, E.M.: Singular Integrals and Differentiability Properties of Functions. Princeton Mathematical Series, No. 30. Princeton University Press, Princeton (1970)

47. Stein, E.M.: Topics in Harmonic Analysis Related to the Littlewood-Paley Theory. Annals of Mathematics Studies, No. 63. Princeton University Press/University of Tokyo Press, Princeton/Tokyo (1970)

48. Strichartz, R.S.: $L^{p}$ harmonic analysis and Radon transforms on the Heisenberg group. J. Funct. Anal. 96(2), 350-406 (1991)

49. Triebel, H.: Spaces of Besov-Hardy-Sobolev type on complete Riemannian manifolds. Ark. Mat. 24(2), 299-337 (1986)

50. Triebel, H.: Characterizations of function spaces on a complete Riemannian manifold with bounded geometry. Math. Nachr. 130, 321-346 (1987)

51. Triebel, H.: Function spaces on Lie groups and on analytic manifolds. In: Function Spaces and Applications (Lund, 1986). Lecture Notes in Mathematics, vol. 1302, pp. 384-396. Springer, Berlin (1988)

52. Triebel, H.: Theory of Function Spaces. II. Monographs in Mathematics, vol. 84. Birkhäuser Verlag, Basel (1992)

53. Triebel, H.: Theory of Function Spaces. Modern Birkhäuser Classics. Birkhäuser/Springer Basel AG, Basel (2010)

54. Triebel, H.: Theory of Function Spaces. IV. Monographs in Mathematics. Birkhäuser Verlag, Basel (2020)

55. Varopoulos, N.T.: Analysis on Lie groups. J. Funct. Anal. 76(2), 346-410 (1988)

56. Varopoulos, N.T., Saloff-Coste, L., Coulhon, T.: Analysis and Geometry on Groups. Cambridge Tracts in Mathematics, vol. 100. Cambridge University Press, Cambridge (1992) 